UNITED STATES DEPARTMENT OF THE INTERIOR GEOLOGICAL SURVEY WATER RESOURCES DIVISION

\title{
SEDIMENT INVESTIGATIONS
}

OF THE

PLATTE RIVER NEAR OVERTON, NEBRASKA

January 1950 to Sept emb er 1953

\section{FOR ADMINISTRATIYE USE ONLY SUBJIECT TO REVISION \\ LPR 197955}

\author{
U, S. Geological Survey \\ 510 Pudge-Guenzel Building \\ Lincoln 6 , rewiaska
}

Prepared as part of a program of the Department of the Interior for development of the Mis souri River basin 


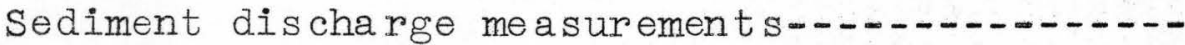

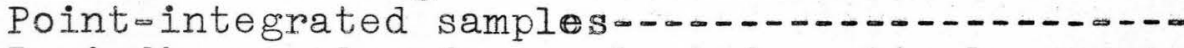

Periodic samples from selected verticals-....Bedmaterial sampleswo..................... Slope determination $\ldots \ldots \ldots+\ldots \ldots \ldots$ Total-load computations $m \ldots \ldots \ldots$

Page

Figure 1. Sketch map showing location of sampling section and profile sections $A$ and $B$, Platte River near Overton, Nebrma....

2. Profile of sections A and B across the channel from staff gages $N=I$ and $N-4-\cdots$

3. Relation of sus pended-sediment discharge to water discharge, January 19, 1950, to september $30,1953 \ldots \ldots$

4. Relation of instantaneous water discharge to suspended sediment finer than 62. microns

5. Distribution of velocity and suspended sediment at stations $45,50,89$, and $189=\ldots \ldots$

6. Distribution of velocity and suspended sediment at stations $260,630,773$, 842 , and $8450-\ldots$

7. Observations of water discharge, suspended-sediment concentration, and stream-bed elevation at station 45-... 14

8. Observations of water discharge, suspended-sediment concentration, and stream-bed elevation at station 845-..- 15

9. Comparison of bed-material and suspended-sediment size analyses-.....

10. Computed sediment discharge plotted against water dis charge 


\section{TABLES}

Table 1. Discharge and particle-size analyses of

Page

suspended sediment................. 18

2. Particle-size analyses of point-and

depth-integrated samples............ 26

3. Observations of water discharge,

suspended sediment, and stream-bed.

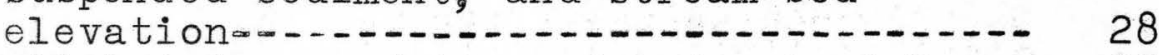

4. Particle-size gradation of bed material-..- 32

5. Periodic observations of water discharge and water-surface slope-............ 35

6. Comparison of total sediment discharge

computed by the modified Einstein procedure with measured sediment

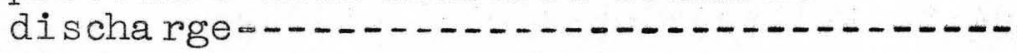


SEDIMENT INVESTIGATIONS OF THE PLATTE RIVER NEAR

OVERTON, NEBRASKA

\section{INT RO DUC TI ON}

This report contains results of sediment-transport investigations on the Platte River near Overton, Nebro, from January 1950 to September 1953. The basic data of suspended-sediment studies, results of bed-material analyses, and determinations of water-surface slopes from staff readings are given.

The data indicate that a reliable determination of suspended sediment, hence total load, is difficult. Because of the nature of the river at the station and the limited scope of the investigations, the suspended-sediment data may not be representative.

The Platte River is characterized by a wide braided channel, a small hydraulic radius, low banks, and a wide flood plain. (See figs. I and 2.) The river bed is composed of coarse to fine sands.

Near Overton, natural flow of the river is controlled or modified by diversions, storage reservoirs, power development, return flow from irrigation, and withdrawals of ground water. A temporary jetty was extended into the river below the bridge during the summer of 1952 as part of commercial 
sand-pumping operations. Beavers carry on active construction in the narrows and shallows, particularly upstream from the sampling section.

Daily fluctuations in water discharge at the gaging station at the bridge are caused by regulation of the flow, mainly from the generation of power by release of water from a reservoir. The water discharge at the station begins increasing about $9: 30 \mathrm{a} . \mathrm{m}_{\text {. }}$, reaches a crest about 2:00 p.m., and then immediately recedes. Weekly water-discharge measurements of alternate high and low stages indicate a daily variation from 200 to more than $1_{g} 000$ cfs。 During spring, summer, and fall, increases in water discharge are also caused by thunderstorm activity in the area.

\section{FIELD STUDIES}

\section{Sediment Discharge Measurements}

A total of 156 sediment discharge measurements were made at or near the gaging station from January 1950 to September 1953. Samples were normally collected with a DH-48 hand sampler. Sediment discharge measurements were usually made at 2-week intervals during periods of high water discharge and less frequently during low water discharge. The water and sediment discharges of each sediment discharge measurement and the particle-size distribution for many of the measurements are show in table 1. 
Samples were collected generally at 20 verticals during high stages and at 10 verticals during low stages. The sampling verticals were spaced in the stream width, which varied from about 300 to 820 feet, either by measurements or by distribution curves of water discharge. Use of the distribution curves probably resulted in small errors in the mean concentration because of the shifting channel.

The maximum measured sediment discharge was 5,870 tons per day for a water discharge of 5,970 cfs, and the minimum was 5.4 tons per day for a water discharge of $166 \mathrm{cfs}$. A relation of suspended-sediment discharge to water discharge is shown in figure 3. The plot illustrates the wide variation of suspended-sediment load or concentration for a given water discharge.

A relation of instantaneous water discharge to suspended sediment finer than 62 microns is shown in figure 4 . Considerable variation in the percentage of the sediment for a given discharge is indicated. However, the figure illustrates a probable trend of about 86 percent finer than 62 microns at a water discharge of 150 cfs to about 20 percent finer at a water discharge of 6,000 cfs。

\section{Point-Integrated Samples}

Point-integrated samples were collected at nine verticals in the cross section with a $P=46$ sampler. The number of 
points in the vertical at which samples were collected ranged from 3 to 5 in depths of water from 1.1 to 5.3 feet. Water velocities at each point were determined by current meter, and depth-integrated samples at each vertical were collected with a $\mathrm{DH}-48$ sampler. (See table 2。) Most of the plotted data outline typical velocity and concentration curves (figs。 5 and 6)。However, as might be expected, some data do not plot near an assumed curve; for example, the concentration observations at the 1.60 and 3.10 depths for station 842 on March 28, 1952.

\section{Periodic Samples From Selected Verticals}

On september 24 and 25, 1952, and April 29 and 30, 1953, suspended-sediment samples were collected simultaneously at stations 45 and 845 about every 2 hours for a period of 30 hours. Stream-bed elevation at the time of collection of each sample was measured by reference to a datum on the bridge railing. Water discharge ranged from 179 to 714 cfs during september 24 to 25, 1952, and from 674 to 1,730 cfs during April 29 to 30, 1953. Sediment samples for the latter period were analyzed also for particle size. Data from the sampling are summarized in table 3 and shown graphically on figures 7 and 8. The graphs illustrate the change in water discharge, concentration, and stream-bed elevation during the period. Figure 8 shows the possibility of 
obtaining erratic concentration data. For example, the concentration was $15 \mathrm{ppm}$ at $9: 50 \mathrm{a} \cdot \mathrm{m}$. and $4,140 \mathrm{ppm}$ at 12:00 m. for a relatively low water discharge of $277 \mathrm{cfs}$.

\section{Bed-Material Samples}

Surface samples of bed material were collected on August $I_{4}$ and september 25, 1952, and on April 16 and september 24, 1953. Five to eight points in the cross section were sampled on each of the days. Also, samples were collected at 5 selected verticals to depths of 0.2 to 3.0 feet from the bed surface. Particle-size gradation for each sample was determined by sieve analysis, and the results are tabulated in table 4. A comparison of bedmaterial and suspended-sediment sizes is given in figure 9. The median size of the bed material was 0.73 millimeter, and the median size of the suspended material was 0.15 millimeter.

\section{Slope Determination}

On May 15, 1952, 4 staff gages were installed on the left bank in a reach of about 2,100 feet and were referred to gage datum. Forty-two observations of slope, usually immediately before or after a sediment discharge measurement, were made during the period from May 15, 1952, to September 24, 1953. The time of the observation, the water 
discharge, and the slope in feet per mile are given in table 5. This table shows that the slope tends to increase with water discharge; thus, the meandering of the flow in the wide channel is decreased。

\section{Total-Load Computations}

Computations of total load were made for a range of water discharge from 242 to $4,170 \mathrm{cfs}$. The results of the computations are shown by figure 10 and table 6. For each determination of total load the cross section was divided into two parts, and a total-load computation was made for each part. The subdivision was necessary because of distinct differences in depth and velocity across the section. For example, on May 15, 1952, a width of 65 feet carried about one-third of the total discharge in a total width of about 700 feet. Depth or velocity or both were used as guides to choose the points of division. A velocity of 1.5 feet per second seems to be about the middle of a transitional zone where movement of bed material of sand size becomes appreciable. For low flows, such as on July 9, 1953, a velocity of 1.5 feet per second was used as a point of division. For normal flows, a depth of 2.5 to 3.0 feet was used as a division point.

For a constant velocity and roughness the unmeasured load varies with the depth. In shallow streams the load passing through the 0.3 foot of depth bel ow the nozzle of 
the sampler is a large percentage of the total load. As the depth increases, the load in the unsampled zone becomes less and less, percentagewise. A plot of unmeasured load (computed total load minus measured load) versus water discharge indicates that unmeasured load increases with water discharge on a slope of about one. If the relation between unmeasured load and water discharge for any one stream is constant, total load can be estimated from measured load with a reasonable degree of accuracy. of course, the relation of unmeasured load to water discharge must first be defined for the range of water discharge.

C。D. Albert

H。 P。 Guy 


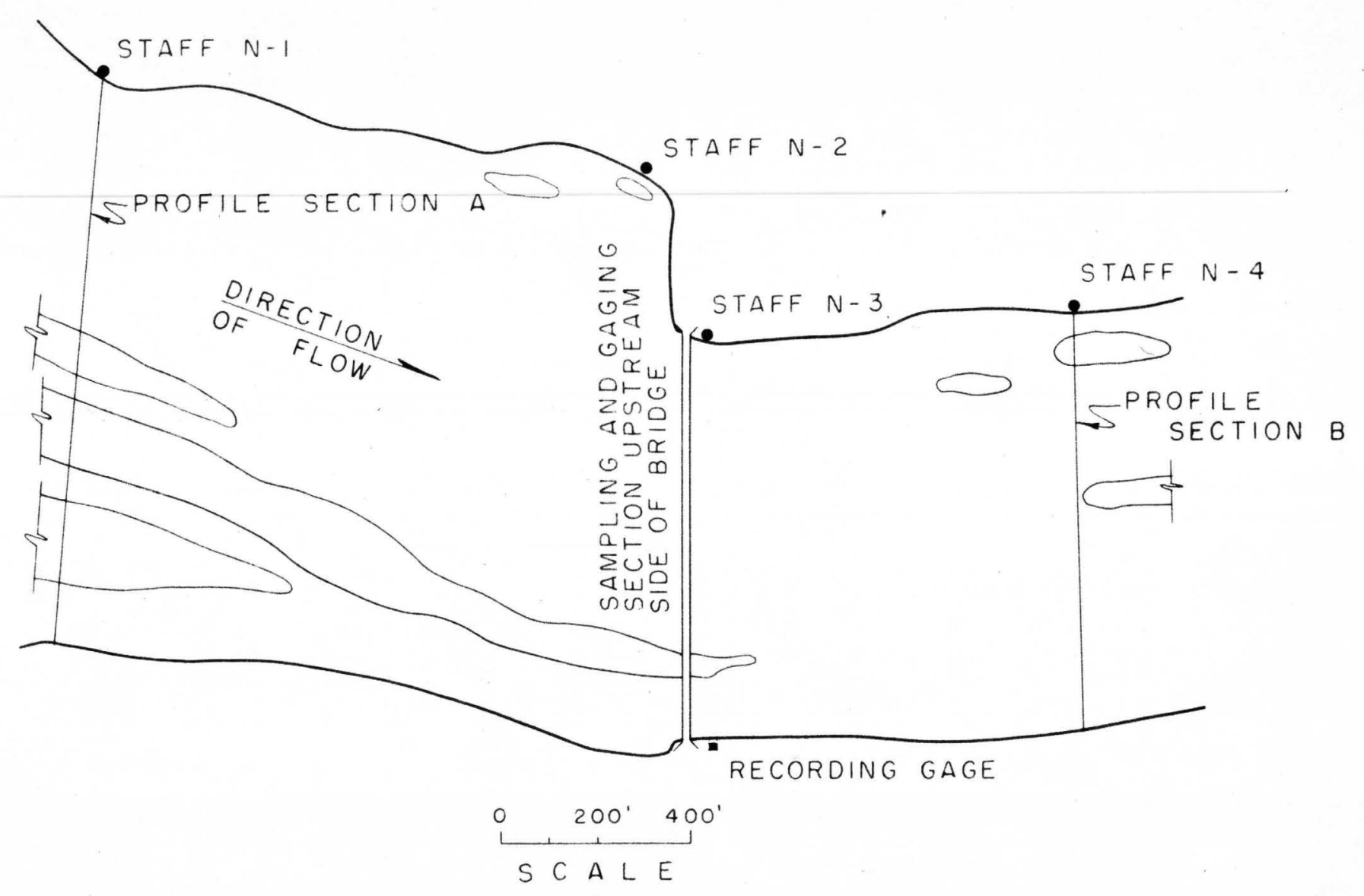

Figure 1.-- Sketch map showing location of sampling section and profile sections $A$ and $B$, Platte River near Overton, Nebr. 


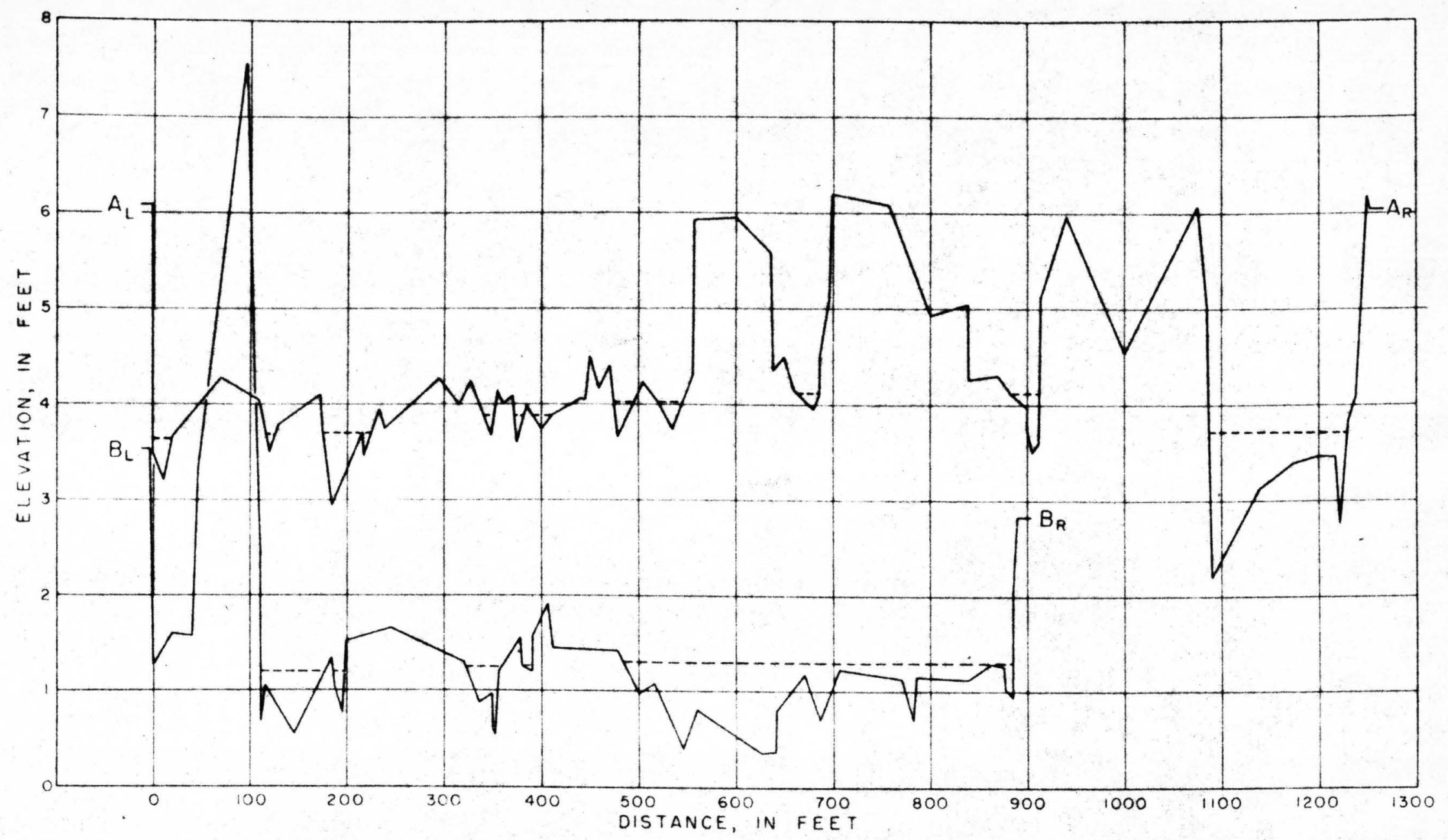
Figure 2.- Proflle of sections 'A and B across the channel from staff gages $N-1$ and $N-4$,
Platte River near Overton, Nebr. 


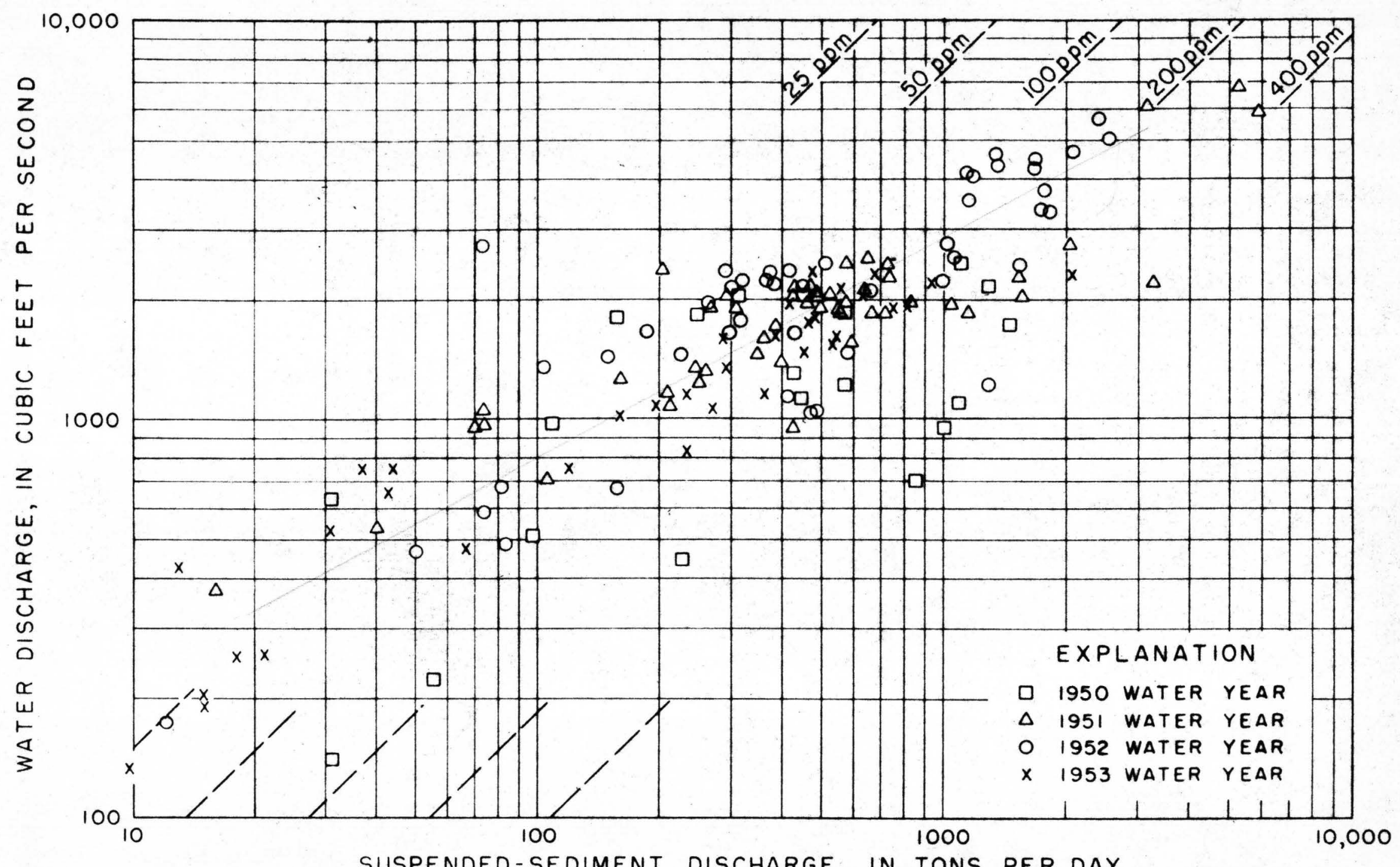

Figure 3.--Relation of suspended-sediment discharge to water discharge, Platte River near Overton, Nebr., January 19,1950, to September 30, 1953. 


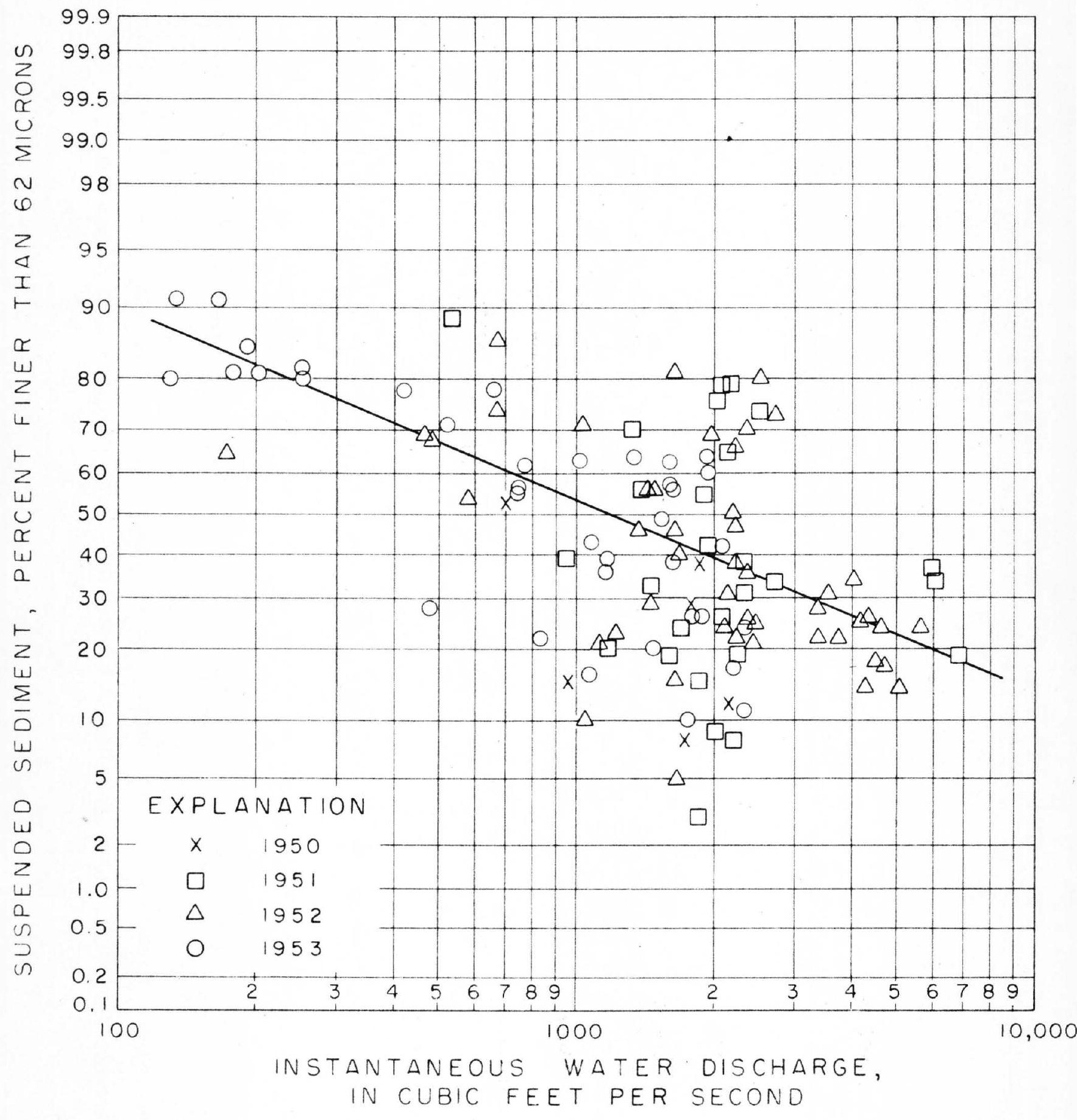

Figure 4.--Relation of instantaneous water discharge to suspended sediment finer than 62 microns, Platte River near Overton, Nebr. 

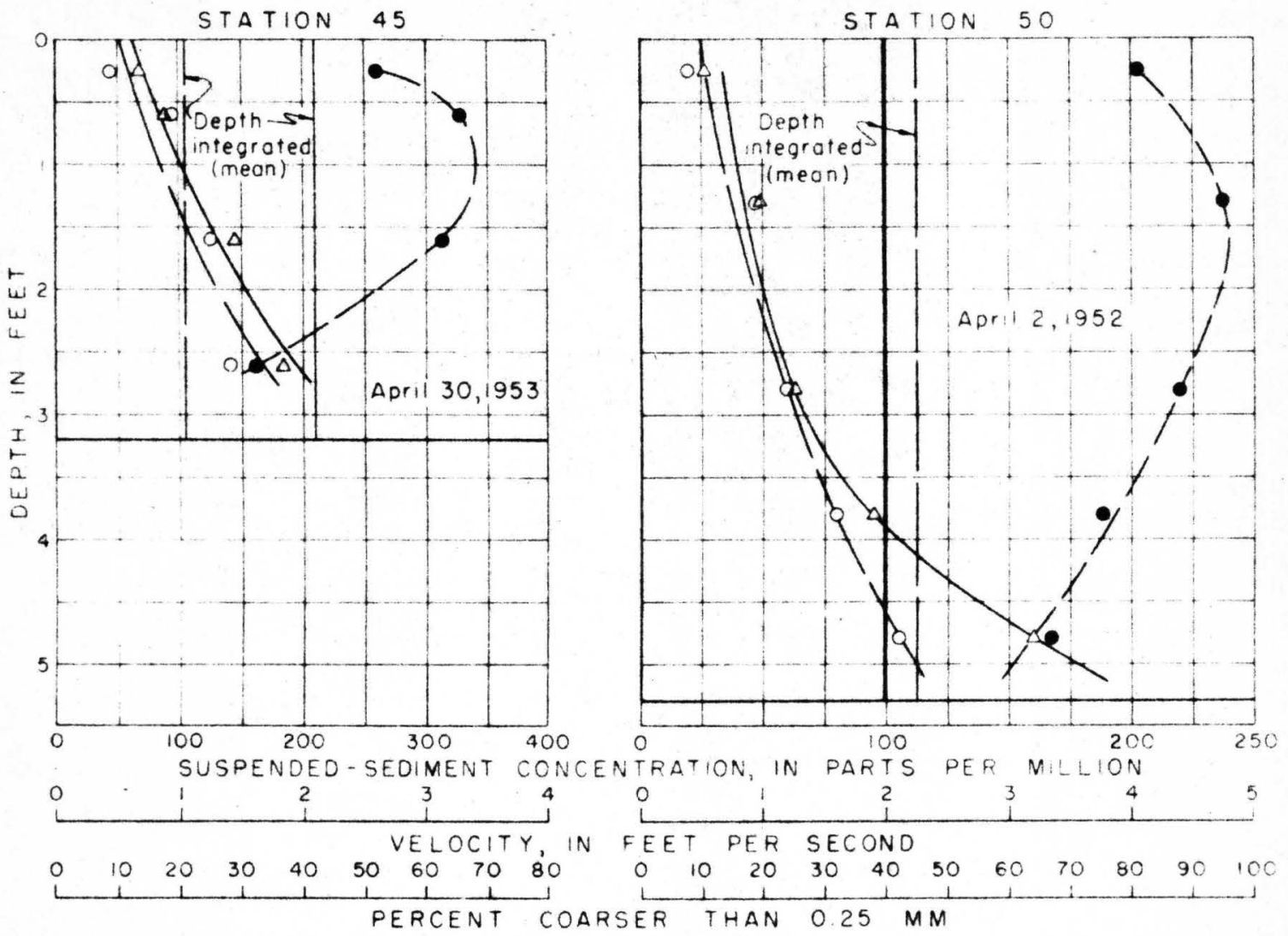

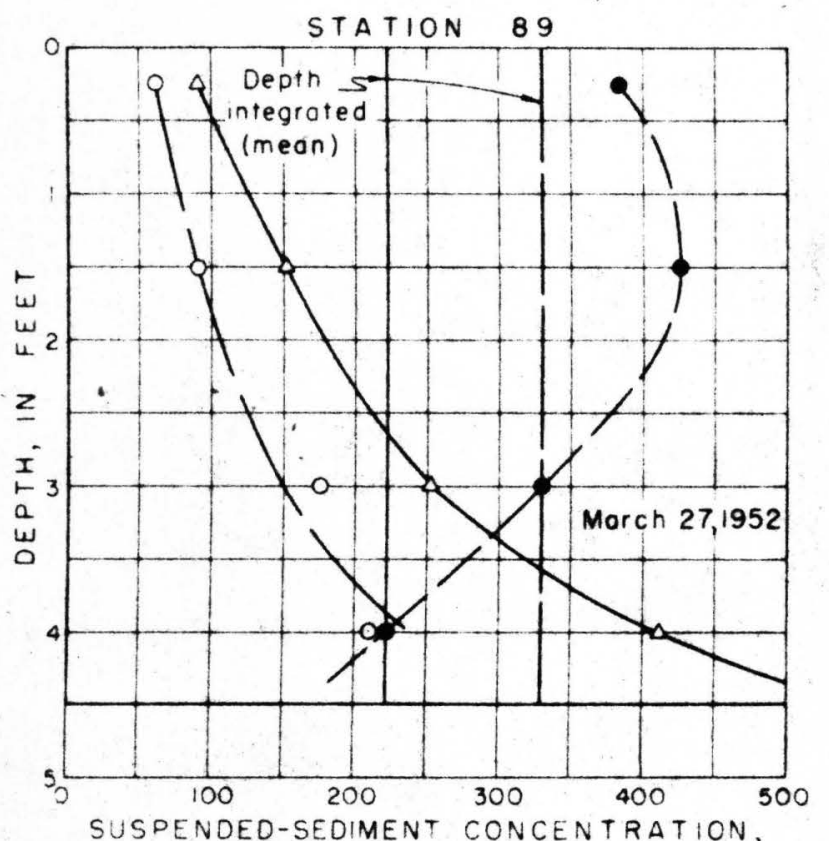

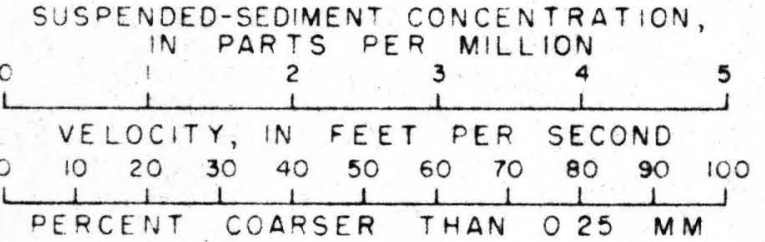
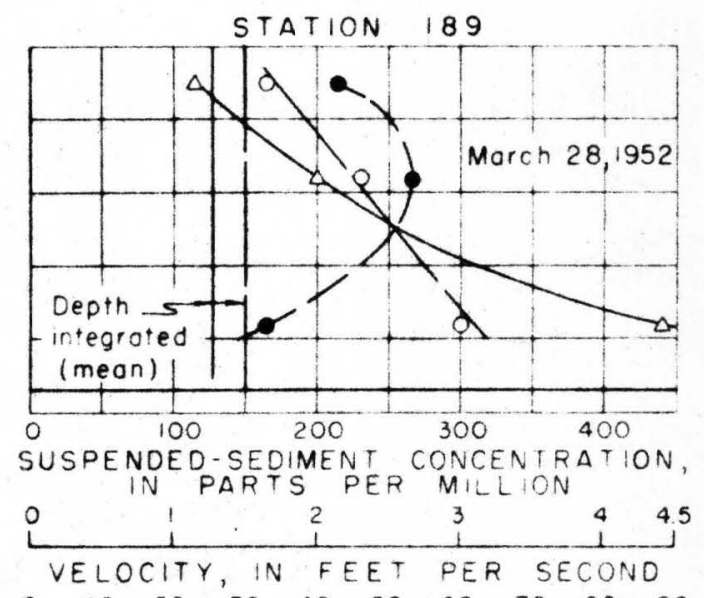

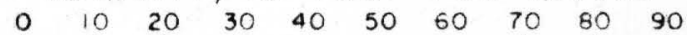

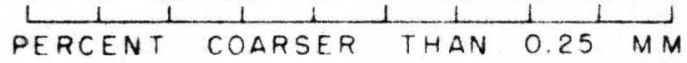

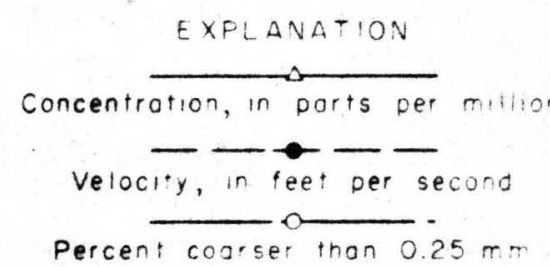

Figure 5 - Distribution of velocity and suspended sediment at stations 45, 50,89, and 189, Platte River near Overton Nebr. 

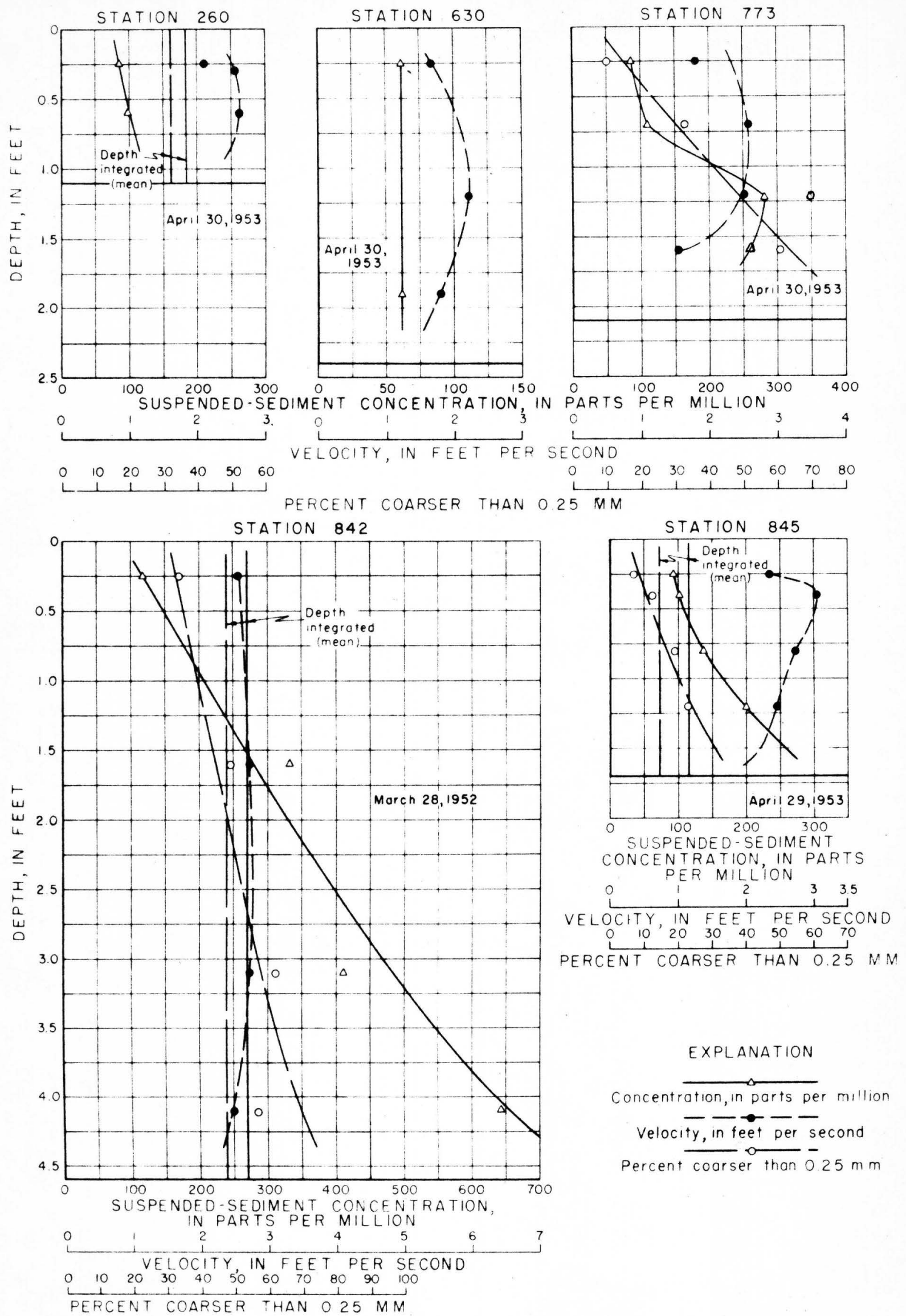

Figure 6 - Distribution of velocity and suspended sediment at stations 260,630,773, 842 , and 845, Platte River near Overton, Nebr. 

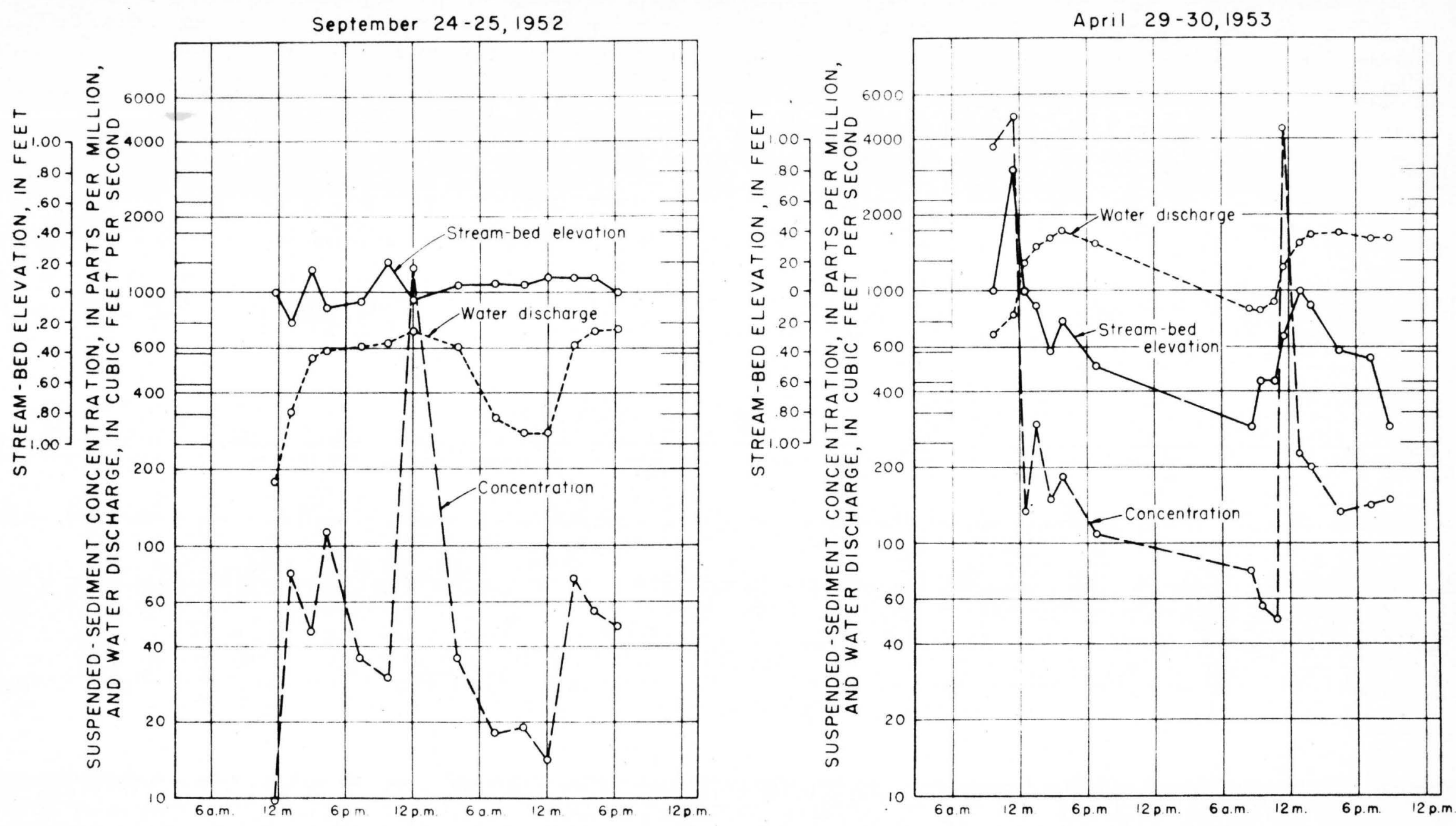

Figure 7.-- Observations of water discharge, suspended-sediment concentration, ond stream-bed elevation at station 45, Platte River near Overton, Nebr. 

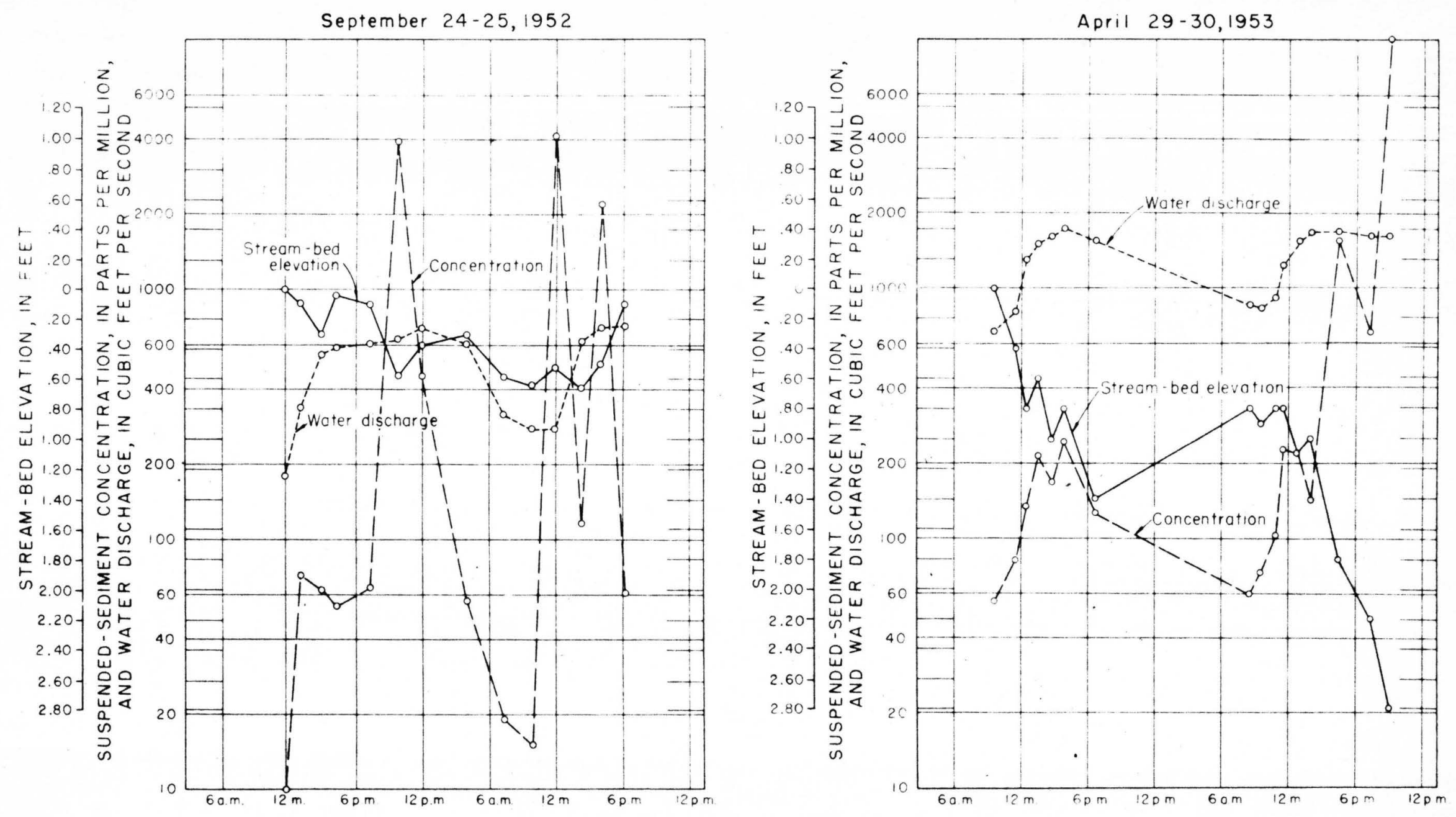

Figure 8 -- Observations of water discharge, suspended-sediment concentration, and stream-bed elevation at station 845, Platte River near Overton, Nebr. 


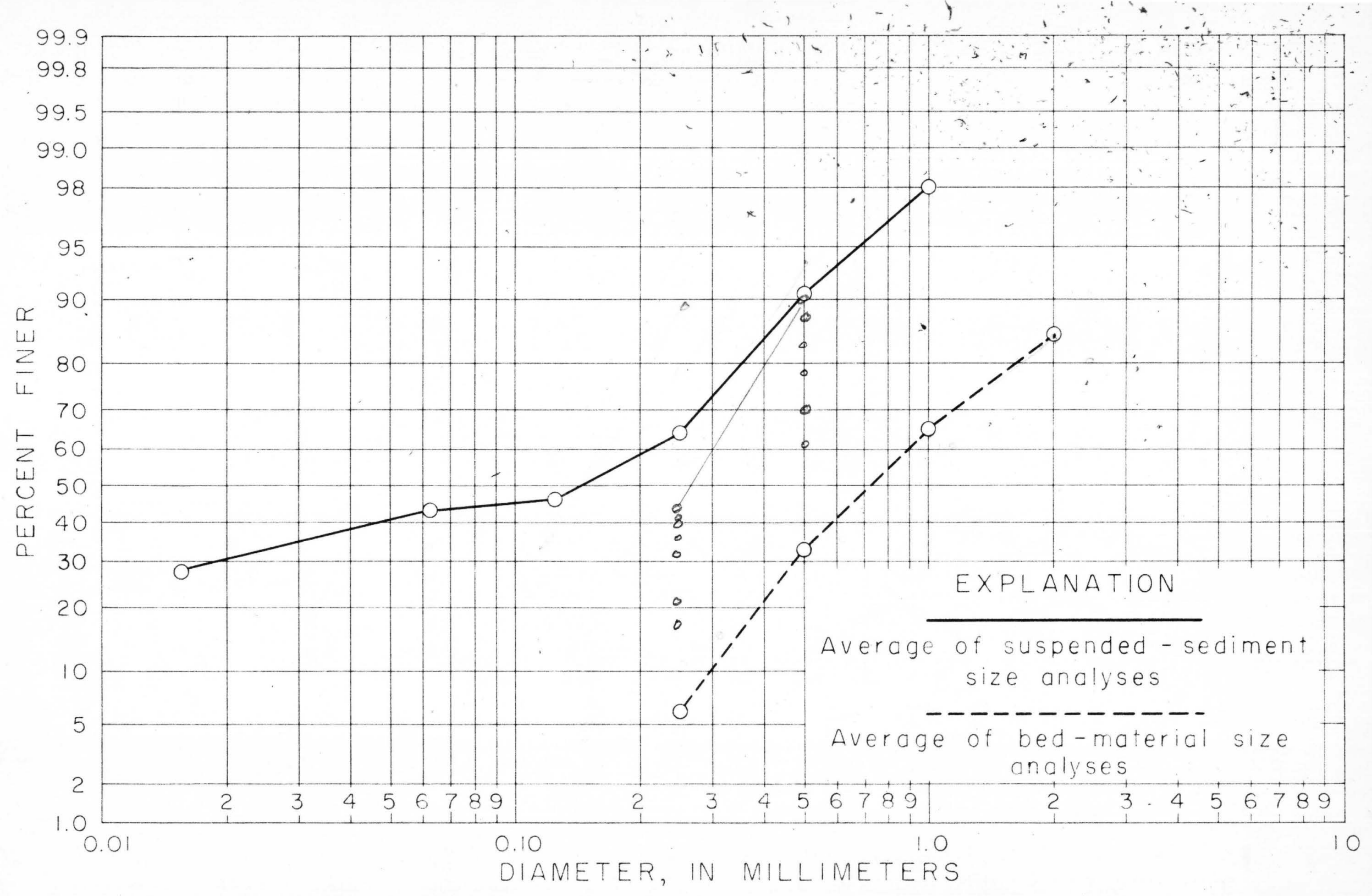

Figure 9 --Comparison of bed-material and súspended-sediment size analyses, Platte River near Overton, Nebr. 


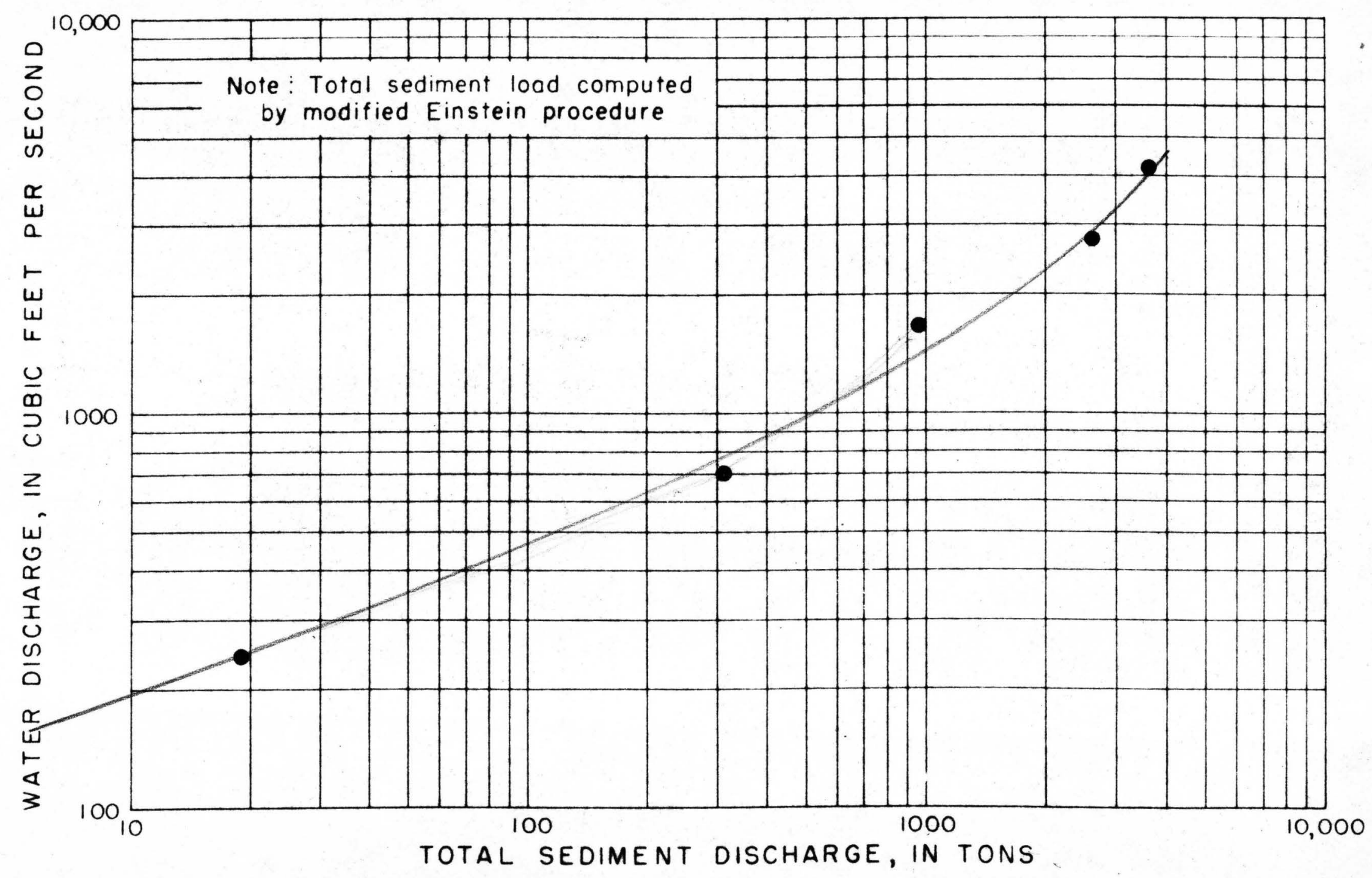

Figure/0.-- Computed sediment discharge plotted against water discharge. 
Table 2.--Discharge and particle-size analyses of suspended sediment, Platte River near Overton, Nebr. Methods of analysis: B, bottom-withdrawal tube; $W$, in distilled water; $S_{2}$ sieve; $C$, chemically dispersed; M, mechanically dispersed; $P$, pipette

\begin{tabular}{|c|c|c|c|c|c|c|c|c|c|c|c|c|c|c|c|}
\hline \multicolumn{2}{|c|}{ Date } & \multicolumn{2}{|c|}{ Time } & $\begin{array}{l}\text { Water } \\
\text { dis- } \\
\text { charge } \\
\text { (cfs) }\end{array}$ & $\begin{array}{l}\text { Concen } \\
\text { tration } \\
\text { (ppm) }\end{array}$ & $\begin{array}{c}\text { Dischargo } \\
\text { (tons per } \\
\text { day) }\end{array}$ & 0.004 & $\frac{\text { pended }}{\text { Perc }}$ & $\begin{array}{l}\text { sedimen } \\
\text { ent fin } \\
\text { size in } \\
0.062\end{array}$ & $\begin{array}{l}\text { er than } \\
\text { millim } \\
0.12 ?\end{array}$ & 0.250 & 0.500 & 1.00 & $\begin{array}{l}\text { Water } \\
\text { temper- } \\
\text { ature } \\
(0 \text { ) })\end{array}$ & $\begin{array}{c}\text { Methods } \\
\text { of } \\
\text { analysis }\end{array}$ \\
\hline \multicolumn{16}{|c|}{1750} \\
\hline $\operatorname{san}$ & 19 & $4: 15$ & $p \cdot m$. & 1,820 & 52 & 256 & $-\infty$ & $-\infty$ & 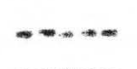 & $\ldots-$ & $-\infty-\infty$ & $-\infty+\infty$ & $-\infty$ & 33 & - n \\
\hline Feb. & $\begin{array}{r}20 \\
1\end{array}$ & $\begin{array}{r}12: 35 \\
6: 30\end{array}$ & $\begin{array}{l}a \cdot m . \\
p=m .\end{array}$ & $\begin{array}{r}1,120 \\
\text { a } 1,800\end{array}$ & $\begin{array}{r}147 \\
32\end{array}$ & $\begin{array}{l}4,4 \\
156\end{array}$ & $\frac{-\infty}{24}$ & 22 & $\begin{array}{c}--. \\
29\end{array}$ & $--\infty=$ & 36 & 40 & 90 & $\begin{array}{l}33 \\
33\end{array}$ & BW \\
\hline & 3 & $5: 30$ & $p \cdot m$. & 2,020 & 57 & 311 & $\ldots$ & $\ldots$ & $-\infty .-$ & $\ldots$ & $-\infty$ & $\ldots$ & $\ldots-$ & 33 & 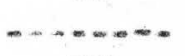 \\
\hline ar. & 1 & $6: 25$ & p.m. & a 2,160 & 222 & 1,290 & 3 & 6 & 11 & 14 & 20 & $\ldots$ & $\cdots$ & 40 & $\mathrm{BW}$ \\
\hline & 2 & $10: 40$ & $a \cdot m$. & 2,020 & 82 & 450 & $-\infty-\infty$ & $-\infty-\infty$ & $\ldots+\infty$ & $\ldots-\cdots$ & $-\infty-\infty$ & $-\infty-\infty$ & $-\infty$ & 42 & $-\infty .-\infty-\infty=$ \\
\hline & 27 & $9: 25$ & 2.7. & 1,220 & 173 & 570 & $-\infty+\infty$ & $----\infty$ & $-\infty-\infty$ & $-\infty$. & $--\infty-\infty$ & $-\infty$ & $\infty-\infty$ & $-\infty-\infty$ & 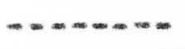 \\
\hline Apr. & 5 & $5: 45$ & p.r. & 2,480 & 268 & 1,790 & $-\cdots-$ & $-\infty-\infty$ & $-\infty-\infty$ & $-\infty-\infty$ & $-\cdots=$ & $-\infty \cdots$ & $\ldots$ & 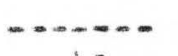 & $-\cdots+\cdots$ \\
\hline & 6 & $10: 30$ & a. $m$. & 2. 1,720 & 313 & 1,450 & 2 & 5 & 8 & 10 & 15 & $\ldots$. & $\cdots$ & 41 & $B W$ \\
\hline May & 3 & $5: 35$ & $\mathrm{p} \cdot \pi \cdot$ & 21,300 & 121 & 425 & $-\infty+\infty$ & $-\infty$ & $--\cdots$ & $\ldots-$ & $-\cdots$ & $-\infty-\infty$ & $\infty$ & 54 & $-\infty-\infty-\infty$ \\
\hline & 4 & $10: 20$ & $\mathrm{a} \cdot \mathrm{m}$ & 634 & 18 & 31 & $\ldots$ & $\ldots .$. & $-\cdots$ & $\ldots \ldots$ & $\ldots$ & - & $-\infty$ & 47 & $\ldots$ \\
\hline & 21 & $6: 90$ & $p \cdot \pi$, & 1,110 & 356 & 1,070 & 60 & 72 & 82 & 90 & 91 & 92 & 95 & 65 & BWCM \\
\hline & 25 & $5: 20$ & $p \cdot m$. & 2958 & 388 & 1,000 & $\ldots+\cdots$ & $-\infty-\infty$ & 25 & 16 & 30 & 58 & 83 & 53 & S \\
\hline June & 15 & $6: 45$ & p.n. & 2701 & $44 ?$ & 846 & $-\infty=\infty$ & $-\infty-\infty$ & 53 & 57 & 71 & 87 & 100 & 88 & $S$ \\
\hline iuly & 6 & $12: 55$ & a.tr. & 2140 & 81 & 31 & $\cdots \cdots$ & $\ldots-\infty$ & 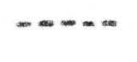 & $\cdots-$ & $-\infty-\infty$ & $\ldots . .$. & $\cdots$ & 75 & $m-\infty+\infty-\infty-\infty$ \\
\hline & 21 & $20: 30$ & a.m. & 446 & 210 & 253 & $\cdots$ & $m-\infty$ & $\ldots$ & $\ldots$ & $\ldots$ & $\ldots$ & $\ldots$ & 73 & $\ldots \ldots$ \\
\hline Aug. & 9 & $5: 40$ & $p . m$. & a 222 & 92 & 55 & $=\ldots$ & $=-\cdots$ & $-\infty$ & $\cdots \cdots$ & $\cdots-$ & $\ldots$ & $\cdots$ & 8 & $\ldots+\cdots$ \\
\hline & 31 & $22: 10$ & p.m. & a 512 & 70 & 97 & $m-\cdots$ & $-\infty-\infty$ & 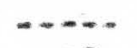 & $-\infty-\infty$ & 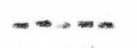 & $-\infty \cdots$ & $-\cdots$ & 76 & $-\rightarrow-\infty+\infty-\infty$ \\
\hline Sept. & 27 & $6: 25$ & p.m. & a 1,850 & $11 \mathrm{~s}$ & 569 & 23 & 30 & 38 & 46 & 75 & $\cdots$ & $-\infty$ & 65 & BNCM \\
\hline & 28 & $11: 05$ & $a, m$. & 977 & 41 & 108 & $\cdots \cdots$ & $-\infty-\infty$ & $=-n-$ & $-\infty-\infty$ & 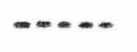 & $m-\cdots$ & $\infty$ & 59 & $-\infty .+\cdots$ \\
\hline
\end{tabular}

a Water discharge measurement made this day. 
Table 1.-. Discharge and particle-size analyses of suspended sediment, Platte River near Overton, Nebr.--Continued

Methods of analysis: B, bottom-withdrawal tube; W, in distilled water; $S$, sieve; C, chemically dispersed; M, mechanically dispersed; $p$, pipette 7

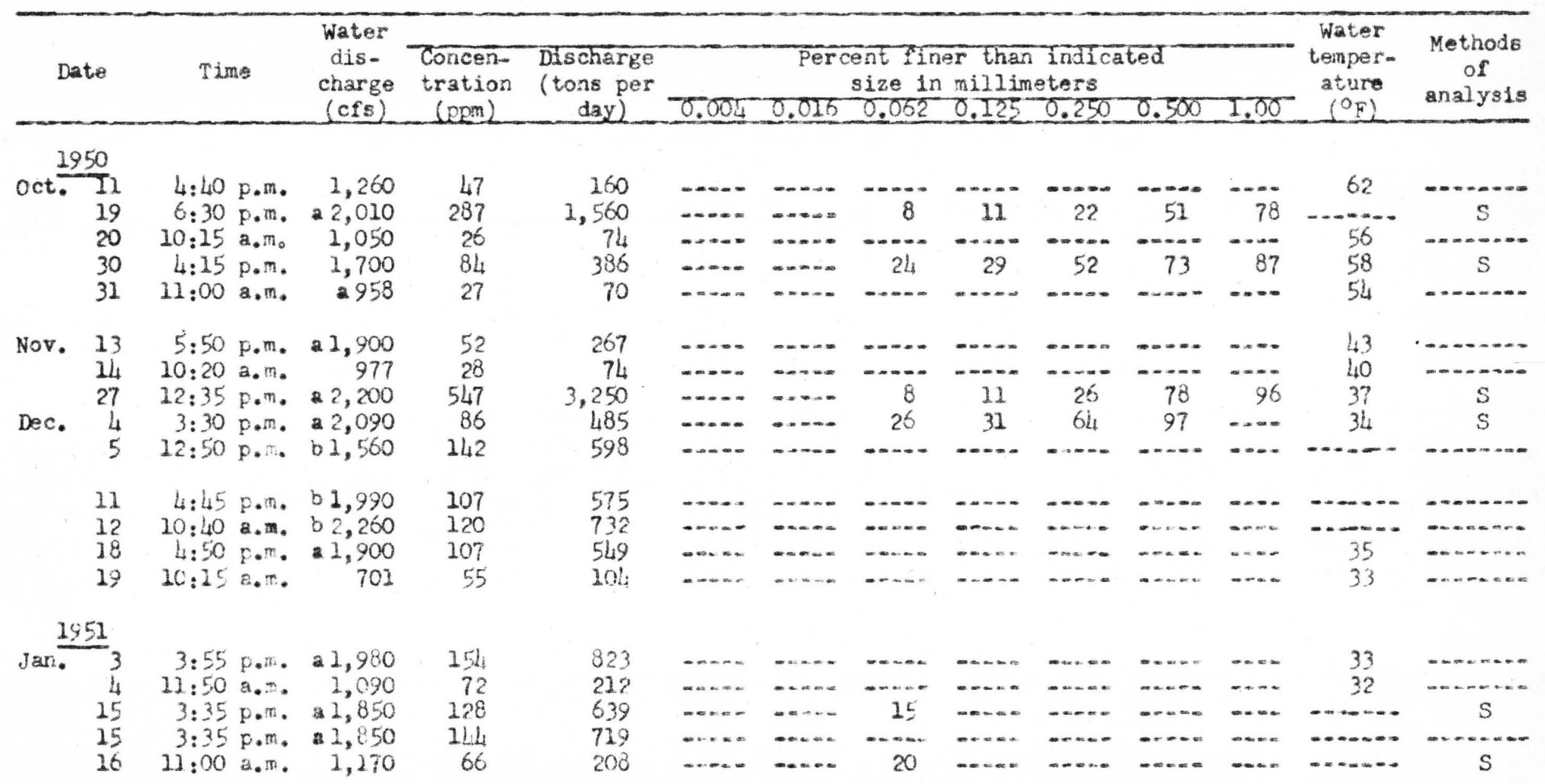

2 Water discharge measurement made this day.

b Mean daily discharge. 
Table 1,--ilscharge and particle-size anaiyses of suspended sediment, Platte Fiver near Cverton, Nebr... Continued

Methods of analysts: B, bottom-whthdrawal tube; $W$, in distilled water; S, steve; C, chenically dispersec; M, mechanically dispersed; $P$, pipette

\begin{tabular}{|c|c|c|c|c|c|c|c|c|c|c|c|c|c|c|c|}
\hline \multicolumn{2}{|c|}{ Dete } & \multicolumn{2}{|c|}{ Time } & $\begin{array}{l}\text { Water } \\
\text { diso } \\
\text { charge } \\
\text { (cfs) }\end{array}$ & $\begin{array}{l}\text { Concen } \\
\text { tration } \\
\text { (ppm) }\end{array}$ & $\begin{array}{c}\text { Gischarge } \\
\text { (tors per } \\
\text { day) }\end{array}$ & 0.004 & $\begin{array}{l}\text { pended } \\
\text { Per }\end{array}$ & $\begin{array}{l}\text { sedine } \\
\text { ent Ii } \\
\text { size i } \\
0.06 ?\end{array}$ & $\begin{array}{l}\text { a tha } \\
\frac{0111}{0.125}\end{array}$ & $\begin{array}{l}\text { indace } \\
\text { eters } \\
0.250\end{array}$ & 0.500 & 1.00 & $\begin{array}{l}\text { Water } \\
\text { teriper- } \\
\text { ature } \\
\left({ }^{\circ} \mathrm{F}\right)\end{array}$ & $\begin{array}{l}\text { Methods } \\
\text { of } \\
\text { anajysis }\end{array}$ \\
\hline \multicolumn{16}{|c|}{1951} \\
\hline & $\begin{array}{l}29 \\
30\end{array}$ & $\begin{array}{r}4: 00 \\
12: 00\end{array}$ & $\begin{array}{l}p \cdot m . \\
m .\end{array}$ & $\begin{array}{r}2,330 \\
\text { b } 1,850\end{array}$ & $\begin{array}{r}32 \\
231\end{array}$ & $\begin{array}{r}201 \\
1,150\end{array}$ & $\ldots$ & $\ldots$ & $\begin{array}{r}31 \\
3\end{array}$ & $\ldots$ & $\ldots$ & $\ldots$ & $\ldots$ & $\begin{array}{l}32 \\
32\end{array}$ & $\begin{array}{l}S \\
S\end{array}$ \\
\hline Feb. & $\begin{array}{l}19 \\
20\end{array}$ & $\begin{array}{l}5: 15 \\
9.55\end{array}$ & p.m. & $\begin{array}{r}2,470 \\
1,200\end{array}$ & $\begin{array}{r}109 \\
76\end{array}$ & $\begin{array}{l}72 \% \\
250\end{array}$ & $\ldots$ & -n.en & $\ldots$ & $m . . .$. & $-m$ & $m a$ & $\ldots$ & 44 & - \\
\hline Mar. & 5 & $5: 00$ & $p \cdot m$. & a 2,460 & 87 & 578 & $\ldots$ & $-\ldots$ & $\ldots$ & $-\infty$. & $\ldots \ldots$ & 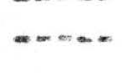 & $\ldots$ & $\ldots$ & - n......... \\
\hline & 6 & $10: 10$ & $a \cdot m$ & 1,340 & 68 & 246 & $\ldots .$. & $\ldots$ & $\ldots \ldots$ & $\ldots n=$ & $\ldots$ & $\ldots$ & $\ldots$ & 33 & $\ldots+\ldots$ \\
\hline & 19 & $3: 45$ & p.m. & a 2,120 & 211 & 635 & $-\cdots+\infty$ & $-\infty=0$ & -..... & n...mes & $--\infty .$. & $-\ldots \infty$ & $=-\infty$ & 39 & mandons \\
\hline & 20 & $10: 45$ & $a . m$. & 1,460 & 88 & 347 & $-x=0$ & $-\infty-\infty$ & 33 & $-\infty-\infty)$ & 66 & 86 & $m-\infty$ & 33 & S \\
\hline Apr. & 2 & $3: 40$ & p.m. & $=2,040$ & 53 & 292 & n.m. & $-\infty .$. & $\ldots$ & -n... & $\cdots$ & $\ldots+\cdots$ & $\ldots \ldots$ & 46 & 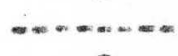 \\
\hline & 3 & $12: 45$ & $2 \cdot m \cdot$ & 1,600 & 83 & 358 & $-\infty \infty$ & $-a+\infty$ & 19 & $-\infty+\cdots$ & $\cdots \infty$ & $\ldots . .$. & $\ldots$ & 48 & $S$ \\
\hline & 16 & $3: 30$ & p. n. & a 1,960 & 87 & 460 & -...... & $--\infty=$ & $-\ldots .$. & $-\ldots-m$ & $\ldots . .$. & $-\cdots+x=$ & - & 49 & 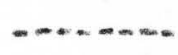 \\
\hline & 17 & $12: 40$ & p.m. & 1,850 & 110 & 549 & $\ldots+\infty$ & 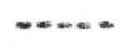 & $-\infty$ & $-\infty \ldots$ & $-\ldots$ & $m-\infty$ & $-\infty$ & 48 & 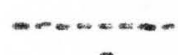 \\
\hline May & 1 & $6: 50$ & p.m. & 2,140 & 110 & 636 & $\ldots$ & $\ldots$ & 65 & $-\ldots-\infty$ & $m$ & $-\infty .$. & $m \infty$ & 57 & S \\
\hline & 17 & $6: 05$ & p.m. & $8.5,970$ & 364 & 5,870 & -... & $\ldots=$ & 37 & $m \cdots$ & $m-\infty$ & 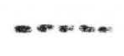 & $\ldots$ & 73 & S \\
\hline & 18 & $9: 00$ & a.m. & 6,020 & 194 & 3,150 & $-\ldots-\infty$ & $-\infty x+\infty$ & 34 & $\ldots+\infty$ & $-m \ldots$ & 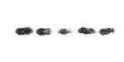 & $-\infty .+\infty$ & 64 & S \\
\hline & 18 & $1: 20$ & $\mathrm{p} \cdot \mathrm{m}$. & 6,870 & 282 & 5,230 & $\ldots$ & $\ldots$ & 19 & $-\infty .+\infty$ & -.... & $\ldots .$. & $-\infty=$ & 64 & S \\
\hline & 24 & $7: 50$ & p. & 22,720 & 276 & 2,030 & $-\infty \ldots$ & $\ldots=m$ & 34 & $\cdots \cdots$ & $-x-\infty$ & $-\ldots=\infty$ & $\ldots$ & 75 & $S$ \\
\hline & 25 & $12: 20$ & P.m. & 2,50 & 95 & 644 & $-\infty-\infty$ & $\cdots+$ & 74 & $\cdots-\infty$ & $-\infty \ldots$ & $-\cdots+\infty$ & $\ldots$ & 74 & $s$ \\
\hline June & 7 & 6.05 & p.m. & a 1,390 & 106 & 398 & $-\ldots$. & $-\infty=\infty$ & 56 & $-\infty-\infty$ & 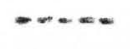 & $m \ldots$ & $-\infty-$ & 69 & $S$ \\
\hline & 21 & $3: 25$ & $p \cdot n$. & 22,060 & 94 & 523 & $-\ldots .-\infty$ & $-\infty$. & 79 & $-\infty \cdots$ & $\ldots$ & $-\infty+\infty$ & $-\infty-\infty$ & $\ldots \ldots$ & S \\
\hline
\end{tabular}

- Water discharge measuremento made this day.

Mean datly discharge. 
Table 1... Discharge and particle-size anzlyses of suspended sediment, Platte River near Overton, Nebr.--Oontinued Methods of analysis: $B$, bottomwithdrawal tube; $W$, in distilled water; $S$, sieve; $C$, chemically dispersed, M,
mechanically dispersed; P, plpette)

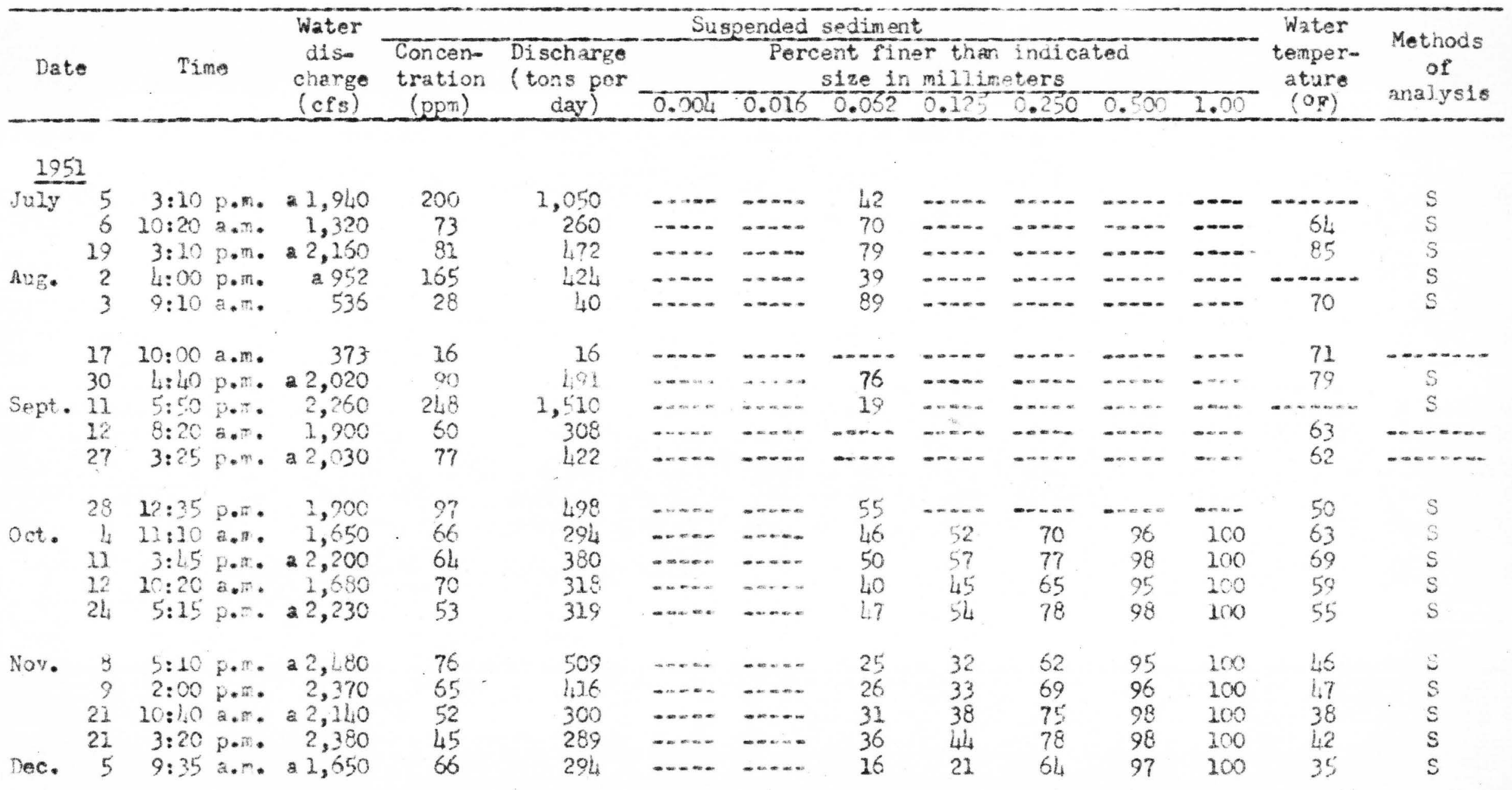

a Water discharge measurement, macie this day. 
Table 1.... Lischarge ard particle-cize analyses of suspended sediment, Platte River near Overton, Nebr...Continued Methods of analysis: B, bottom-wthdrawal tube; W, in distilled water; $S_{3}$ sieve; C, chemically dispersed; M, mechariculy dispersed; P, pipete?

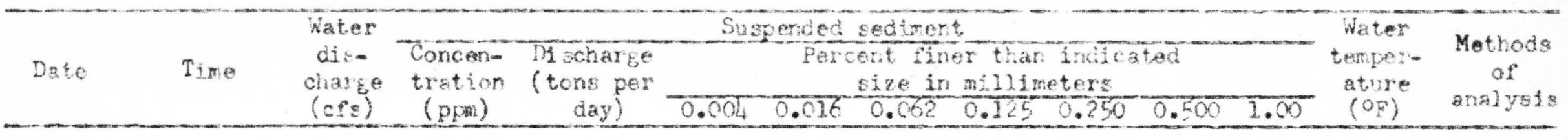

1951

\begin{tabular}{|c|c|c|c|c|c|c|c|c|c|c|c|c|}
\hline $\begin{array}{r}5 \\
28 \\
29\end{array}$ & $\begin{array}{r}2: 25 \mathrm{p} . \mathrm{m} . \\
2: 00 \mathrm{p} . \mathrm{m} . \\
11: 15 \mathrm{a} . \mathrm{m} .\end{array}$ & $\begin{array}{r}2,230 \\
\text { b } 1,040 \\
\text { b } 1,130\end{array}$ & $\begin{array}{r}62 \\
172 \\
237\end{array}$ & $\begin{array}{l}367 \\
483 \\
418\end{array}$ & $\ldots$ & 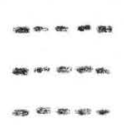 & $\begin{array}{l}22 \\
10 \\
21\end{array}$ & $\begin{array}{l}28 \\
12 \\
22\end{array}$ & $\begin{array}{l}64 \\
16 \\
28\end{array}$ & $\begin{array}{l}98 \\
55 \\
54\end{array}$ & $\begin{array}{r}100 \\
90 \\
91\end{array}$ & $\begin{array}{r}39 \\
\ldots \ldots\end{array}$ \\
\hline
\end{tabular}

1952

\begin{tabular}{|c|c|c|c|c|c|c|c|c|c|c|c|c|c|c|c|}
\hline \multirow{3}{*}{ Jan. } & 11 & $11: 00$ & $a . m$. & 22,100 & 116 & 658 & $-\ldots+\ldots$ & $m$ & 24 & 26 & 39 & 78 & 95 & $-m-\cdots$ & $S$ \\
\hline & 11 & $4: 15$ & $\mathrm{p} \cdot \mathrm{m}$. & $b 2,210$ & 72 & 430 & $\ldots . .$. & $\ldots$ & 38 & 41 & 64 & 96 & 100 & $\ldots$ & S \\
\hline & 31 & $3: 15$ & p.r. & 23,350 & 188 & 1,700 & $\ldots \ldots$ & $\ldots$ & 28 & 37 & 69 & 98 & 200 & $\ldots \ldots$ & S \\
\hline \multirow[t]{4}{*}{ Feb. } & 1 & $9: 05$ & $\mathrm{a}, \mathrm{m}$. & b 3,380 & 206 & 1,880 & 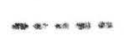 & $-\cdots$ & 22 & 28 & 61 & 97 & 100 & 32 & $S$ \\
\hline & $1 !$ & $12: 00$ & $a \cdot m$. & 3,550 & 120 & 1,150 & $-m-\infty$ & $-\ldots+\infty$ & 31 & 38 & 68 & 98 & 100 & $-\cdots+\infty$ & S \\
\hline & $\mathrm{H}_{4}$ & $4: 45$ & p.m. & a 4,050 & 107 & 1,170 & -nar. & $\ldots$ & 34 & 42 & 70 & 98 & 100 & w..... & $S$ \\
\hline & 22 & $3: 50$ & p.m. & 4,340 & 115 & 1,350 & 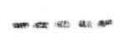 & 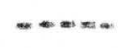 & 26 & 35 & 72 & 98 & 100 & $m-\infty$. & S \\
\hline \multirow[t]{7}{*}{ Mar. } & 5 & $5: 30$ & p.m. & 24,700 & 165 & 2,090 & $-\ldots \ldots$ & $\ldots . .$. & 17 & 22 & 56 & 90 & 96 & 33 & $S$ \\
\hline & 6 & $10: 50$ & $a . m$ & 4,270 & 145 & 1,670 & $\ldots \ldots$ & -n. & 14 & 15 & 26 & 90 & 99 & 33 & S \\
\hline & 29 & $7: 15$ & p.r. & a 3,770 & 173 & 1,760 & $-\infty-\infty$ & $\cdots=\infty$ & 22 & 28 & 50 & 88 & 96 & 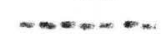 & S \\
\hline & 20 & $11: 00$ & a.m. & 4,510 & 137 & 1,570 & $-\ldots . .$. & $-\ldots . .$. & 18 & 26 & 56 & 91 & 100 & 44 & $\omega^{\prime}$ \\
\hline & 20 & $2: 10$ & p.re. & 4,610 & $10 ?$ & 1,330 & $\ldots$ & $\cdots \cdots$ & $2 L$ & 32 & 58 & 90 & 99 & $\omega$ & S \\
\hline & 27 & $1: 20$ & $p . m$. & 5,670 & 156 & 2,390 & $-\cdots$ & $\ldots$ & 24 & 31 & 63 & 94 & 99 & $45^{\circ}$ & S \\
\hline & 28 & $9: 20$ & a.. & a 5,030 & 186 & 2,530 & $-\ldots \ldots$ & n... & Ih & 29 & 52 & 90 & 98 & 41 & S \\
\hline Apre & 2 & $1: 20$ & p.w. & a. 4,170 & 101 & $1,11,0$ & $\ldots$ & $-\infty \infty$ & 25 & 31 & 63 & 93 & 100 & 53 & S \\
\hline
\end{tabular}

a Water discharge measurement made this day.

b Mean daily discharge. 
Table 1... Discharge and particle-tze analyses of suspended sediment, Platte River near Overton, Nebr... Continaed

Method of analysiv: B, bottonathdrawal tube; W, in distilled water; Sieve; C, chemically dispersed; M, mecharically dispersed; $P$, pipette?

\begin{tabular}{|c|c|c|c|c|c|c|c|c|c|c|c|c|c|c|c|}
\hline \multicolumn{2}{|c|}{ Date } & \multicolumn{2}{|c|}{ Time } & $\begin{array}{c}\text { Water } \\
\text { df } \\
\text { charga } \\
\text { (cfs) }\end{array}$ & $\begin{array}{l}\text { Concer } \\
\text { tration } \\
\text { (ppm) }\end{array}$ & $\begin{array}{c}\text { Discharge } \\
\text { (tons per } \\
\text { day) }\end{array}$ & 0.504 & $\begin{array}{r}\text { ended } \\
\text { Perce } \\
0.016\end{array}$ & $\begin{array}{l}\text { sediment } \\
\text { ont fine } \\
\frac{\text { sze in }}{0.062}\end{array}$ & $\begin{array}{l}r \text { than } \\
\text { mi11 ime } \\
0.125\end{array}$ & $\begin{array}{l}\text { indicat } \\
\text { ters } \\
0.250\end{array}$ & 0.500 & & $\begin{array}{l}\text { Water } \\
\text { temper- } \\
\text { ature } \\
\left(\mathrm{O}_{\mathrm{g}}\right)\end{array}$ & $\begin{array}{c}\text { Methods } \\
\text { of } \\
\text { analysis }\end{array}$ \\
\hline \multicolumn{16}{|c|}{1952} \\
\hline Aps. & $\begin{array}{r}16 \\
16 \\
29 \\
2 \\
11\end{array}$ & $\begin{array}{l}9: 20 \\
3: 10 \\
1: 45 \\
5: 10 \\
5: 00\end{array}$ & $\begin{array}{l}\text { a.m. } \\
p \cdot m \cdot \\
p \cdot m \cdot \\
p \cdot m . \\
p \cdot m .\end{array}$ & $\begin{array}{r}1,460 \\
\text { a } 2,720 \\
2,430 \\
2,370 \\
1,980\end{array}$ & $\begin{array}{r}57 \\
10 \\
232 \\
58 \\
49\end{array}$ & $\begin{array}{r}225 \\
73 \\
1,520 \\
371 \\
262\end{array}$ & 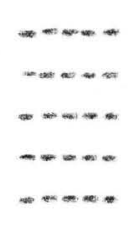 & $\begin{array}{c}\ldots+\ldots \\
\ldots \ldots \\
\ldots+\infty \\
\ldots \ldots\end{array}$ & $\begin{array}{l}29 \\
34 \\
21 \\
70 \\
69\end{array}$ & $\begin{array}{l}32 \\
38 \\
22 \\
74 \\
72\end{array}$ & $\begin{array}{l}54 \\
59 \\
29 \\
87 \\
83\end{array}$ & $\begin{array}{l}92 \\
92 \\
68 \\
99 \\
98\end{array}$ & $\begin{array}{r}100 \\
98 \\
92 \\
100 \\
100\end{array}$ & $\begin{array}{l}47 \\
50 \\
63 \\
66 \\
71\end{array}$ & $\begin{array}{l}S \\
S \\
S \\
S \\
S\end{array}$ \\
\hline & $\begin{array}{l}15 \\
15 \\
15 \\
28 \\
28\end{array}$ & $\begin{array}{r}8: 15 \\
11: 45 \\
4: 10 \\
9: 30 \\
1: 35\end{array}$ & $\begin{array}{l}\text { a.m. } \\
\text { a.m. } \\
\text { p.m. } \\
\text { a.m. }\end{array}$ & $\begin{array}{r}1,370 \\
1,440 \\
21,580 \\
2,230 \\
22,540\end{array}$ & $\begin{array}{r}28 \\
38 \\
41 \\
164 \\
155\end{array}$ & $\begin{array}{r}104 \\
148 \\
186 \\
988 \\
1,060\end{array}$ & $\frac{-\ldots}{33}$ & $\begin{array}{l}1 \\
42 \\
42\end{array}$ & $\begin{array}{l}46 \\
56 \\
5 \\
66 \\
80\end{array}$ & $\begin{array}{l}50 \\
58 \\
15 \\
69 \\
84\end{array}$ & $\begin{array}{l}68 \\
75 \\
51 \\
78 \\
89\end{array}$ & $\begin{array}{l}95 \\
98 \\
90 \\
96 \\
97\end{array}$ & $\begin{array}{l}100 \\
100 \\
100 \\
100 \\
100\end{array}$ & $\begin{array}{l}60 \\
72 \\
67 \\
63 \\
72\end{array}$ & $\begin{array}{c}S \\
S \\
\text { SPWCM } \\
\text { SPWCM } \\
\text { SPACM }\end{array}$ \\
\hline July & $\begin{array}{l}12 \\
13 \\
26 \\
16 \\
17\end{array}$ & $\begin{array}{l}4: 00 \\
9: 25 \\
3: 30 \\
3: 35 \\
9: 30\end{array}$ & $\begin{array}{l}\text { p.m. } \\
\text { a.m. } \\
\text { p.m. } \\
p \cdot m \cdot \\
a \cdot n .\end{array}$ & $\begin{array}{r}2,750 \\
1,550 \\
1,480 \\
1,220 \\
3,468\end{array}$ & $\begin{array}{r}136 \\
96 \\
145 \\
393 \\
40\end{array}$ & $\begin{array}{r}1,010 \\
4.28 \\
579 \\
1,290 \\
50\end{array}$ & $\begin{array}{c}32 \\
56 \\
-\ldots \\
12 \\
\ldots \ldots\end{array}$ & $\begin{array}{c}48 \\
69 \\
\ldots-\ldots \\
16 \\
\ldots \ldots-\end{array}$ & $\begin{array}{l}73 \\
81 \\
56 \\
23 \\
69\end{array}$ & $\begin{array}{l}75 \\
83 \\
60 \\
25 \\
80\end{array}$ & $\begin{array}{l}86 \\
90 \\
73 \\
31 \\
84\end{array}$ & $\begin{array}{r}98 \\
99 \\
96 \\
63 \\
100\end{array}$ & $\begin{array}{r}100 \\
100 \\
99 \\
91 \\
-\ldots\end{array}$ & $\begin{array}{c}8 \mathrm{~L} \\
7 \mathrm{p} \\
-\ldots-\ldots\end{array}$ & $\begin{array}{l}\text { SPWCM } \\
\text { SPWCM } \\
S \\
\text { SBWCM } \\
\text { S }\end{array}$ \\
\hline Sept. & $\begin{array}{l}31 \\
13 \\
14 \\
28 \\
24\end{array}$ & $\begin{array}{r}9: 40 \\
3: 10 \\
11: 25 \\
6: 35 \\
4: 00\end{array}$ & 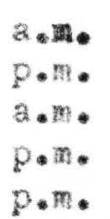 & $\begin{array}{r}173 \\
1,030 \\
.674 \\
489 \\
584\end{array}$ & $\begin{array}{r}25 \\
172 \\
87 \\
63 \\
47\end{array}$ & $\begin{array}{r}12 \\
476 \\
158 \\
83 \\
74\end{array}$ & 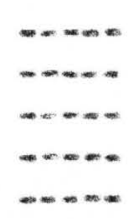 & $\begin{array}{l}\ldots \ldots \\
\ldots-\ldots \\
\ldots \ldots-\infty \\
\ldots-\infty\end{array}$ & $\begin{array}{l}65 \\
71 \\
86 \\
68 \\
54\end{array}$ & $\begin{array}{l}72 \\
89 \\
71 \\
56\end{array}$ & $\begin{array}{l}80 \\
93 \\
84 \\
70\end{array}$ & $\begin{array}{r}94 \\
100 \\
97 \\
100\end{array}$ & $\begin{array}{c}100 \\
100 \\
\ldots \ldots\end{array}$ & $\begin{array}{c}70 \\
85 \\
79 \\
75 \\
-\cdots\end{array}$ & $\begin{array}{l}S \\
S \\
S \\
S \\
S\end{array}$ \\
\hline
\end{tabular}

2 Water discharge measurement made this day. 
Table 1..-Discharge and particle-size analyses of suspended sediment, Platte River near Overtor, Nebr... Continued Methode of analysis: $B$, bottom-witharaal tube; W, in distilled water; $S_{2}$ sieve; C, chemically dispersed; M,
mechanically dispersed; $P$, pipettef

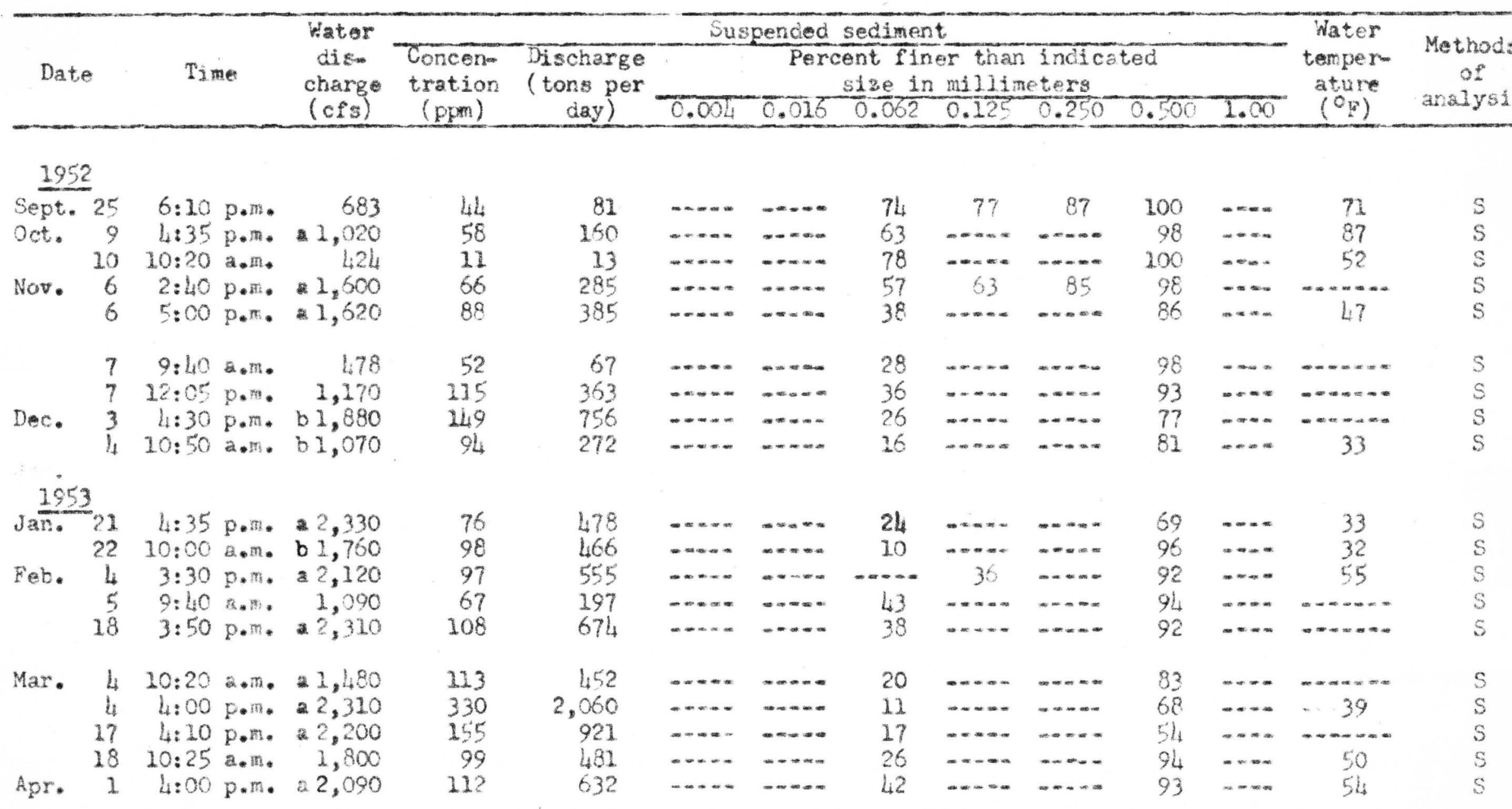

a Water discharge measurement made this ay.

b Mean daily discharge. 
Table 1.--Discharge and particle-size analyses of suspended sediment, Platte River near Overton, Nebr.--Continued Methods of analysis: $B$, bottom-withdrawal tube; W, in distilled water; S, sieve; C, chenically dispersed; M, mechanically dispersed; $P$, plpettel

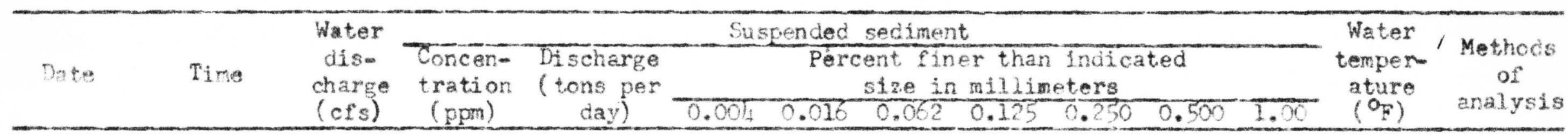

\begin{tabular}{|c|c|c|c|c|c|}
\hline \multirow[t]{7}{*}{ Apr. } & 2 & $10: 00$ & a.m. & 755 & 19 \\
\hline & 15 & $4: 25$ & p.m. & a 1,930 & 154 \\
\hline & 16 & $10: 05$ & $\approx, n$. & 755 & 59 \\
\hline & 28 & $4: 45$ & 2.7 & 1,530 & 129 \\
\hline & 29 & $5: 15$ & p. 70 & 1,600 & 82 \\
\hline & 30 & $10: 10$ & a.n. & a 833 & $104_{4}$ \\
\hline & 30 & $7: 20$ & $p \cdot v$. & a 1,520 & 124 \\
\hline \multirow[t]{4}{*}{ May } & $1 / 4$ & $3: 15$ & p. an & a 1,960 & 77 \\
\hline & 15 & $9: 50$ & 2. & 772 & 21 \\
\hline & 28 & $3: 20$ & $p * m_{*}$ & 21,340 & 81 \\
\hline & 29 & $9: 30$ & $a, n$ & 660 & 24 \\
\hline \multirow[t]{4}{*}{ June } & 11 & $3: 50$ & p.r. & 1,170 & 74 \\
\hline & 12 & $8: 05$ & $a_{0} x_{s}$ & 524 & 22 \\
\hline & 24 & $2: 30$ & p.n. & 2166 & 12 \\
\hline & 25 & $7: 30$ & a.m. & 179 & 16 \\
\hline \multirow[t]{2}{*}{ July } & 9 & $3: 30$ & $p . \eta_{\text {. }}$ & 2253 & 27 \\
\hline & 10 & $8: 40$ & a.m. & $2 !$ & 32 \\
\hline \multirow[t]{2}{*}{ Aug. } & 20 & $3: 50$ & p. $\pi$. & 2135 & 26 \\
\hline & 21 & $9: 30$ & 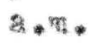 & 130 & 28 \\
\hline \multirow[t]{2}{*}{ Sept. } & $2 \%$ & $9: 15$ & a. 7 . & 2. 203 & 28 \\
\hline & 24 & $3: 10$ & $p_{0} \pi$. & $=192$ & 30 \\
\hline
\end{tabular}

\begin{tabular}{|c|c|c|}
\hline 37 & $\ldots \ldots$ & $-\infty=\infty$ \\
\hline 802 & D & $=\infty-\infty$ \\
\hline 120 & $\ldots \infty$ & 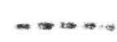 \\
\hline 533 & $\ldots+\infty$ & $-\infty+\infty$ \\
\hline 354 & $\rightarrow \infty \infty$ & $-\ldots$ \\
\hline 235 & $\ldots$ & $\ldots \ldots$ \\
\hline 42 & $-\infty \ldots$ & $\rightarrow \infty x \rightarrow \infty$ \\
\hline $40 ?$ & $\ldots$ & $\cdots+\infty$ \\
\hline 44 & $\cdots \infty$ & $\ldots \ldots$ \\
\hline 293 & $\ldots \ldots$ & $=\ldots$ \\
\hline
\end{tabular}

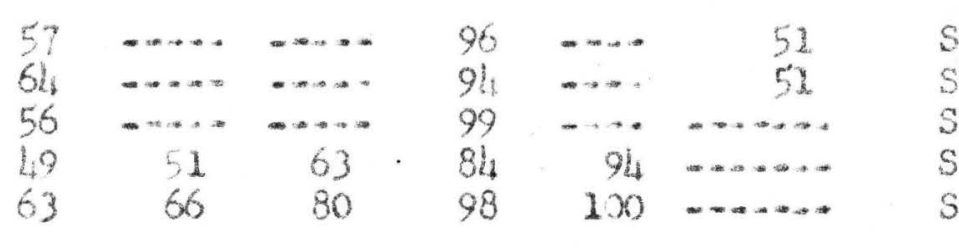

$43 \quad \cdots \cdots+\ldots \ldots+79$

$234 \quad \ldots \ldots+\cdots \cdots \quad 3$

$32, \ldots \ldots+\cdots \cdots \quad 7$

$7.7 \quad \ldots . .+\cdots \infty$

22

$23 \quad 35$

$\begin{array}{cccc}85 & 100 & \ldots \ldots \ldots & S \\ 96 & 100 & \ldots \ldots \ldots & S \\ 92 & \ldots & 62 & S \\ 99 & \ldots & 51 & S \\ 99 & \ldots & 80 & S\end{array}$

$+\infty+\infty+\infty$

$\cdots+\infty$

$\ldots+\ldots$

$\begin{array}{cccc}99 & \ldots & 69 & S \\ 74 & \ldots & 8 ? & S \\ 99 & \ldots & 72 & S \\ 99 & \cdots & 89 & S \\ 99 & \cdots & \cdots & 5\end{array}$

$\begin{array}{ccc}18 & \cdots \cdots & \cdots \cdots \\ 21 & \cdots \cdots & \cdots \cdots \\ 9.5 & \cdots \infty \cdots & \cdots \cdots \\ 9.8 & \cdots \cdots & \cdots \cdots \\ 15 & \cdots \cdots \cdots & \cdots \cdots \\ 15 & \cdots \cdots & \cdots \cdots\end{array}$

82
80
91
80
81
85

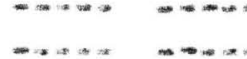

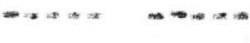

$+\infty+\infty+\infty+\infty+\infty$

$\cdots$

$\begin{array}{ll}84 & 92 \\ 83 & 90 \\ 92 & 96 \\ 85 & 93 \\ 84 & 92 \\ 86 & 87\end{array}$

100
98
100
100
100
92

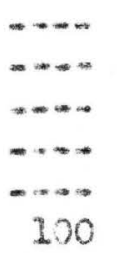

$\begin{array}{cc}75 & S \\ \ldots-\infty & S \\ 69 & S \\ \cdots \infty & S \\ 76 & S\end{array}$

a Water discharge measurement made this day. 
Table 2...Particle-size analyses of point and depth-integrated samples, Platte River near Overton, Nebr. Method of analysis: Sieve]

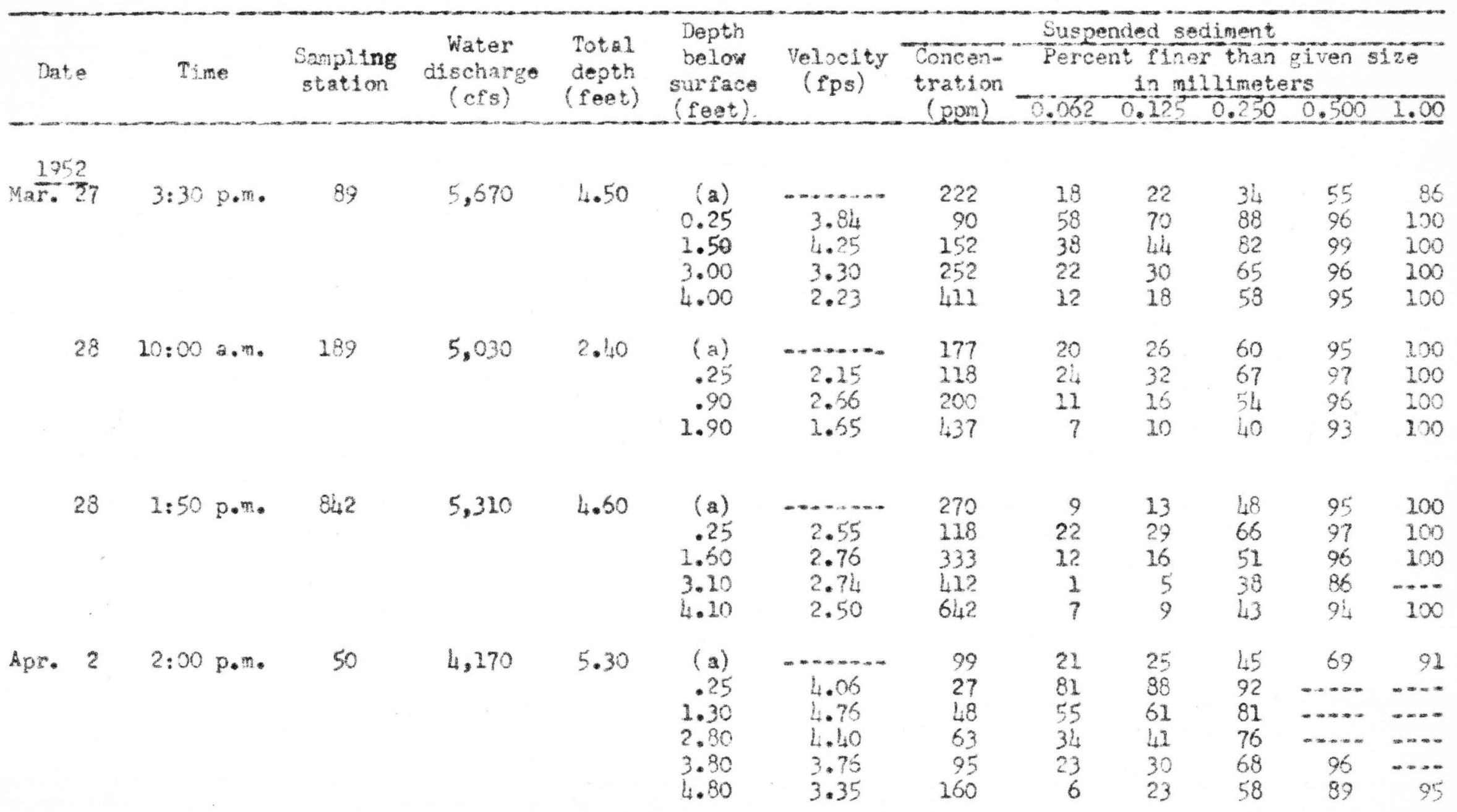

a Deptholintegrated sample. 
Table 2.-.Particle-size analyses of point and depth-integrated samples, Platte River near Overton, Nebr.- Continued Method of analysis: Sieve]

\begin{tabular}{|c|c|c|c|c|c|c|c|c|c|c|c|c|}
\hline Date & Tine & $\begin{array}{l}\text { Sampling } \\
\text { station }\end{array}$ & $\begin{array}{c}\text { Water } \\
\text { discharge } \\
\text { (c.s) }\end{array}$ & $\begin{array}{l}\text { Total } \\
\text { depth } \\
\text { (feet) }\end{array}$ & $\begin{array}{l}\text { Depth } \\
\text { below } \\
\text { surface } \\
\text { (foet) }\end{array}$ & $\begin{array}{c}\text { Velocity } \\
\text { (fps) }\end{array}$ & $\begin{array}{l}\text { Concan } \\
\text { tration } \\
\text { (ppm) }\end{array}$ & $\begin{array}{r}\text { Susper } \\
\text { Percer } \\
-0.062 \\
\end{array}$ & $\begin{array}{l}\text { ded sed } \\
t \sin 9 \\
\sin \sin \\
0.125\end{array}$ & $\begin{array}{l}\frac{\text { diment }}{x} \text { Enan } \\
\text { Dimate } \\
\frac{0.250}{2}\end{array}$ & $\begin{array}{l}\text { Tiven siz } \\
0.500 \\
\end{array}$ & 1.00 \\
\hline$\frac{1953}{A p r \cdot 29}$ & 4:30 p.m. & 845 & 1,680 & 2.70 & $\begin{array}{r}(a) \\
0.25 \\
.40 \\
.80 \\
1.20\end{array}$ & $\begin{array}{l}2.35 \\
3.03 \\
2.71 \\
2.45\end{array}$ & $\begin{array}{r}116 \\
93 \\
102 \\
137 \\
200\end{array}$ & $\begin{array}{l}40 \\
59 \\
47 \\
34 \\
33\end{array}$ & $\begin{array}{l}54 \\
73 \\
61 \\
46 \\
39\end{array}$ & $\begin{array}{r}71 \\
1 \quad 86 \\
75 \\
62 \\
54\end{array}$ & $\begin{array}{r}94 \\
100 \\
90 \\
90 \\
93\end{array}$ & $\begin{array}{r}1.00 \\
100 \\
98 \\
100\end{array}$ \\
\hline 30 & $3: 30 \mathrm{p.t.}$ & 773 & 1,730 & 2.10 & $\begin{array}{r}.25 \\
.70 \\
1.20 \\
1.60\end{array}$ & $\begin{array}{l}2.79 \\
2.56 \\
2.52 \\
2.55\end{array}$ & $\begin{array}{l}86 \\
121 \\
283 \\
258\end{array}$ & $\begin{array}{l}5 ? \\
41 \\
16 \\
21\end{array}$ & $\begin{array}{l}76 \\
54 \\
20 \\
25\end{array}$ & $\begin{array}{l}90 \\
67 \\
30 \\
39\end{array}$ & $\begin{array}{r}100 \\
97 \\
78 \\
96\end{array}$ & $\begin{array}{r}100 \\
93 \\
100\end{array}$ \\
\hline 30 & 4:00 p.m. & 45 & 1,700 & 3.10 & $\begin{array}{r}(\mathrm{a}) \\
.25 \\
.60 \\
1.60 \\
2.60\end{array}$ & $\begin{array}{l}2.63 \\
3.28 \\
3.14 \\
1.63\end{array}$ & $\begin{array}{r}110 \\
67 \\
89 \\
145 \\
184\end{array}$ & $\begin{array}{l}41 \\
32 \\
32 \\
20 \\
14\end{array}$ & $\begin{array}{l}45 \\
55 \\
48 \\
35 \\
30\end{array}$ & $\begin{array}{l}79 \\
91 \\
81 \\
75 \\
72\end{array}$ & $\begin{array}{l}100 \\
100 \\
100 \\
98 \\
99\end{array}$ & $\begin{array}{l}\ldots . . \\
\ldots \ldots \\
100 \\
100\end{array}$ \\
\hline 30 & $4: 50 \mathrm{p.m}$ & 260 & 1,680 & 1.10 & $\begin{array}{l}.7) \\
.25 \\
.30 \\
.60\end{array}$ & $\begin{array}{l}2.11 \\
2.56 \\
2.53\end{array}$ & $\begin{array}{r}\frac{182}{85} \\
98\end{array}$ & $\begin{array}{c}35 \\
84 \\
-\cdots \\
72\end{array}$ & $\begin{array}{c}40 \\
90 \\
-\ldots \\
80\end{array}$ & $\begin{array}{c}68 \\
100 \\
-\infty-0 \\
99\end{array}$ & $\begin{array}{c}89 \\
\cdots \cdots \\
\cdots \cdots\end{array}$ & $\begin{array}{r}97 \\
\ldots-\ldots \\
\ldots\end{array}$ \\
\hline 30 & $5: 15 \mathrm{p} . \mathrm{m}$ & 630 & 1,680 & 2.140 & $\begin{array}{l}(a) \\
.25 \\
1.20 \\
1.90\end{array}$ & $\begin{array}{l}1.68 \\
2.21 \\
1.90\end{array}$ & $\begin{array}{c}62 \\
62 \\
63\end{array}$ & $\begin{array}{c}94 \\
100 \\
93\end{array}$ & $\begin{array}{c}100 \\
\ldots \ldots \\
200\end{array}$ & $\ldots$ & 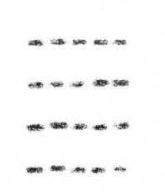 & $\ldots$ \\
\hline
\end{tabular}

a Depth-integrated sample. 
Table 3.-mobservtions of water discharge, suspended sedment, and strean-bed elevation, Plette River near Overton, Nebr.

$$
\text { Method of analysis: Sieve? }
$$

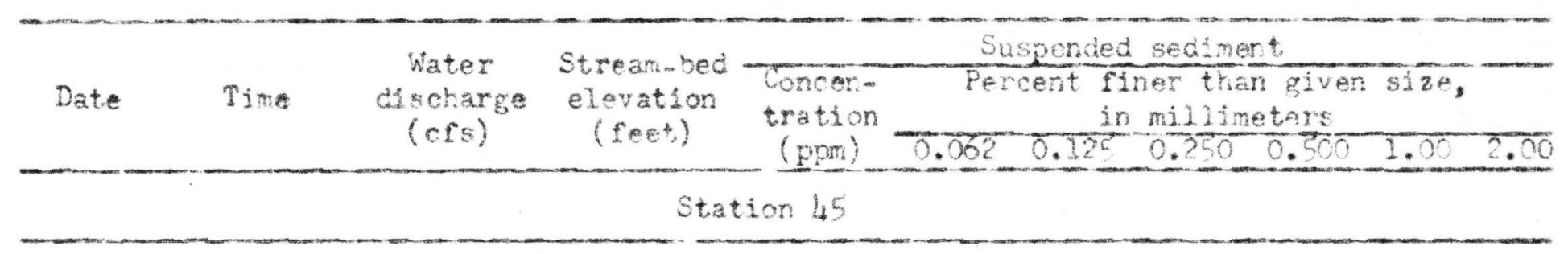

$\begin{array}{rrr}1952 & & \\ \text { Sept. } 24 & 11: 40 \mathrm{a} \cdot \mathrm{m} \cdot & 179 \\ 24 & 1: 05 \mathrm{p} \cdot \mathrm{m} \cdot & 337 \\ 24 & 3: 00 \mathrm{p} \cdot \mathrm{m} \cdot & 548 \\ 24 & 4: 20 \mathrm{p} \cdot \mathrm{m} \cdot & 584 \\ 24 & 7: 20 \mathrm{p} \cdot \mathrm{m} \cdot & 608 \\ & & \\ 24 & 9: 45 \mathrm{p} \cdot \mathrm{m} \cdot & 634 \\ 24 & 17: 00 \mathrm{p} \cdot \mathrm{m} \cdot & 701 \\ 25 & 4: 00 \mathrm{~m} \cdot \mathrm{m} \cdot & 508 \\ 25 & 7: 20 \mathrm{a} \cdot \mathrm{m} \cdot & 318 \\ 25 & 9: 50 \mathrm{a} \cdot \mathrm{m} \cdot & 277 \\ 25 & 12: 00 \mathrm{~m} \cdot & 277 \\ 25 & 2: 20 \mathrm{p} \cdot \mathrm{m} \cdot & 620 \\ 25 & 4: 10 \mathrm{p} \cdot \mathrm{m} \cdot & 701 \\ 25 & 6: 15 \mathrm{p} \cdot \mathrm{m} \cdot & 714\end{array}$

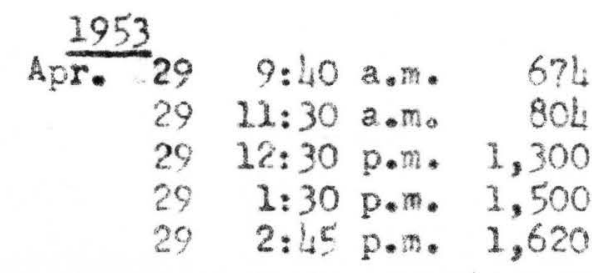

$$
\begin{array}{r}
0.00 \\
-.20 \\
+.15 \\
. .10 \\
. .06 \\
+.20 \\
. .05 \\
. .05 \\
. .06 \\
+.05 \\
+.10 \\
+.10 \\
. .10 \\
.00
\end{array}
$$

7
78
46
113
36
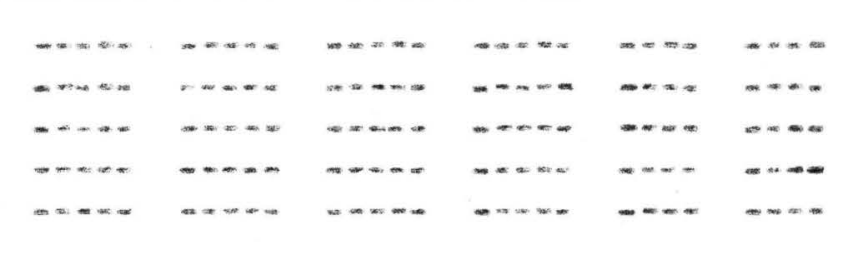

$\begin{array}{lr}+.20 & 30 \\ . .05 & 1.250\end{array}$
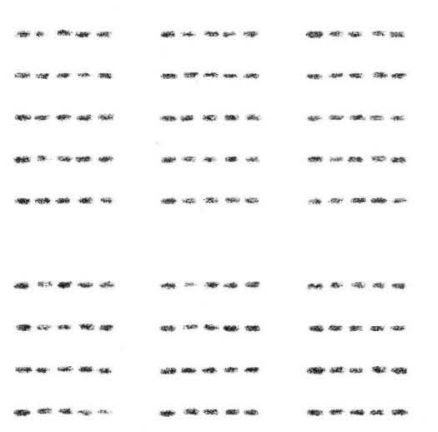

-n....

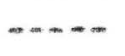

$m \infty$

36
18

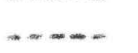

$\ldots . .$.

n... nas

a......-

$\ldots+\cdots$

14
74
55
48

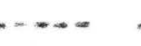

$m+\infty-\infty$

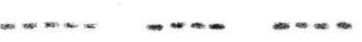

-....

$+\cdots$

$\infty \cdots+$

-nso

-... -...

-

.00

.00
+.80

.00

$-.10$

3,750
4,960
133
297
150

$\begin{array}{cc}0 & 1 \\ -\ldots & 0 \\ 27 & 41 \\ 12 & 16 \\ 25 & 33\end{array}$

1
5
66
33
59

$\begin{array}{rrr}20 & 72 & 96 \\ 53 & 98 & 100 \\ 96 & 100 & \ldots . \\ 86 & 100 & \ldots . . \\ 96 & 100 & \ldots . .\end{array}$


Table 3.--Observations of water discharge, cuspended sediment, and stream-ted elevation, Platte River near Overton, Nebr.--Continued

Method of analysis: Sieve]

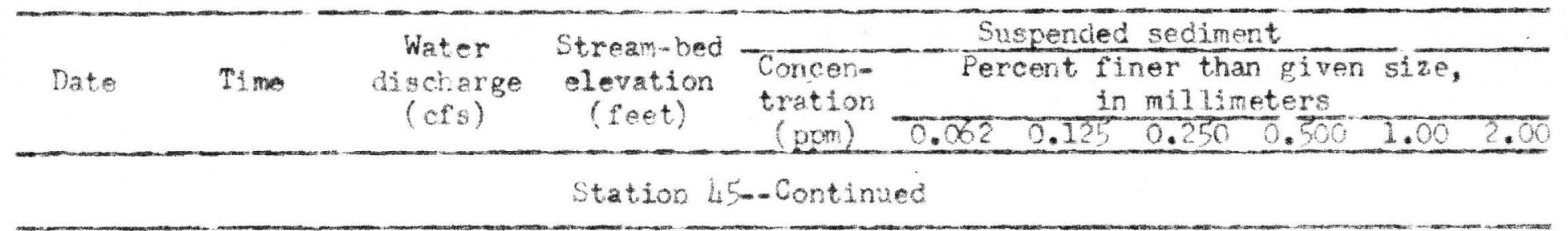

\begin{tabular}{|c|c|c|c|c|c|c|c|c|c|c|c|c|}
\hline Apr. & $\begin{array}{l}29 \\
29 \\
30 \\
30 \\
30\end{array}$ & $\begin{array}{r}3: 45 \\
6: 45 \\
8: 30 \\
9: 30 \\
10: 45\end{array}$ & $\begin{array}{l}\text { p.m. } \\
\text { p.m. } \\
\text { a.m. } \\
\text { a.m. } \\
\text { a.m. }\end{array}$ & $\begin{array}{r}1,730 \\
2,550 \\
854 \\
838 \\
904\end{array}$ & $\begin{array}{r}-0.20 \\
-.50 \\
-.90 \\
-.60 \\
-.60\end{array}$ & $\begin{array}{r}184 \\
109 \\
78 \\
56 \\
50\end{array}$ & $\begin{array}{l}24 \\
30 \\
30 \\
17 \\
38\end{array}$ & $\begin{array}{l}31 \\
36 \\
39 \\
23 \\
45\end{array}$ & $\begin{array}{l}58 \\
60 \\
74 \\
66 \\
80\end{array}$ & $\begin{array}{r}94 \\
91 \\
95 \\
97 \\
100\end{array}$ & $\begin{array}{r}98 \\
100 \\
100 \\
100 \\
\ldots\end{array}$ & $\begin{array}{r}100 \\
-\ldots \\
\ldots-\infty \\
-\ldots\end{array}$ \\
\hline & $\begin{array}{l}30 \\
30 \\
30 \\
30 \\
30\end{array}$ & $\begin{array}{r}11: 30 \\
1: 00 \\
2: 00 \\
4: 30 \\
7: 20\end{array}$ & $\begin{array}{l}\text { a.m. } \\
\text { p.m. } \\
\text { p.m. } \\
\text { p.m. } \\
\text { p.m. }\end{array}$ & $\begin{array}{l}1,2140 \\
1,550 \\
1,680 \\
1,700 \\
1,620\end{array}$ & $\begin{array}{r}-.30 \\
.00 \\
-.10 \\
-.40 \\
-.45\end{array}$ & $\begin{array}{r}4,110 \\
227 \\
200 \\
132 \\
112\end{array}$ & $\begin{array}{l}1 \\
24 \\
30 \\
44 \\
34\end{array}$ & $\begin{array}{l}1 \\
31 \\
34 \\
50 \\
42\end{array}$ & $\begin{array}{l}4 \\
61 \\
68 \\
80 \\
66\end{array}$ & $\begin{array}{l}32 \\
95 \\
95 \\
99 \\
96\end{array}$ & $\begin{array}{l}75 \\
100 \\
100 \\
100 \\
100\end{array}$ & $\begin{array}{r}97 \\
-\infty-\cdots \\
-\infty-\infty \\
-\infty-\infty\end{array}$ \\
\hline & 30 & $9: 00$ & p.m. & 1,620 & -.90 & 149 & 35 & 39 & 69 & 96 & 100 & $-\cdots$ \\
\hline
\end{tabular}

\begin{tabular}{|c|c|c|c|c|c|c|c|c|c|c|c|}
\hline Sept.2L & $11: 40$ & an. & 179 & 0.00 & 10 & $\ldots \ldots$ & $-\infty \ldots$ & 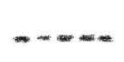 & $-\infty-\infty$ & $-\infty$ & $-\infty$ \\
\hline 24 & $1: 05$ & p.m. & 337 & -.10 & 72 & $-\infty \ldots$ & $-\infty-\infty-$ & $-m-\infty$ & $-\infty-\infty$ & $-\infty-\infty$ & $\ldots$ \\
\hline 24 & $3: 00$ & p.m. & 548 & -30 & 63 & $\ldots+\infty-\infty$ & $-\infty-\infty$ & $-\infty-\infty$ & 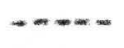 & $-\infty$ & $\cdots$ \\
\hline 24 & $4: 20$ & p.th. & 584 & -.04 & 54 & 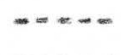 & $\cdots$ & $-\infty-\infty$ & $-\infty-\infty$ & $-\infty-\infty$ & 5 \\
\hline 24 & $7: 20$ & pom. & 608 & -.10 & 64 & $\ldots+\infty$ & $-\cdots-\infty$ & $-\cdots$ & $-\infty \cdots$ & $-\infty-$ & 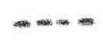 \\
\hline
\end{tabular}


Table 3.-. Cbservations of water dischame, suspended-sediment, and stream-bed elevation, Platte Kiver near Overton, Nebr..-Continued

\section{Method of analysis: Sieve7}

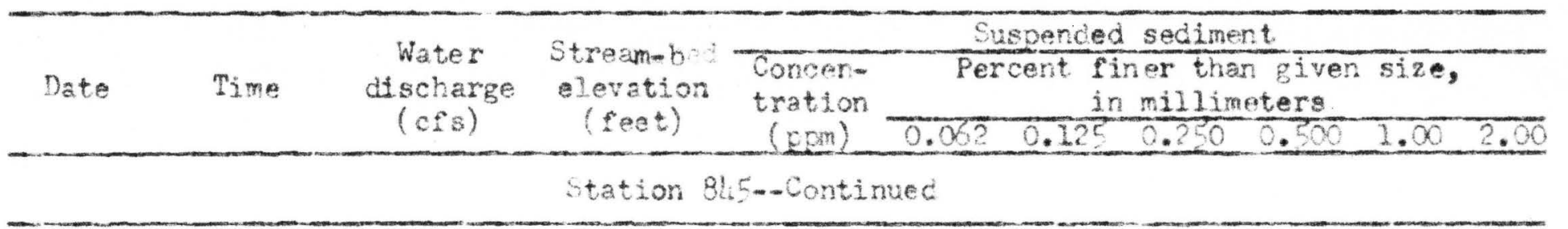

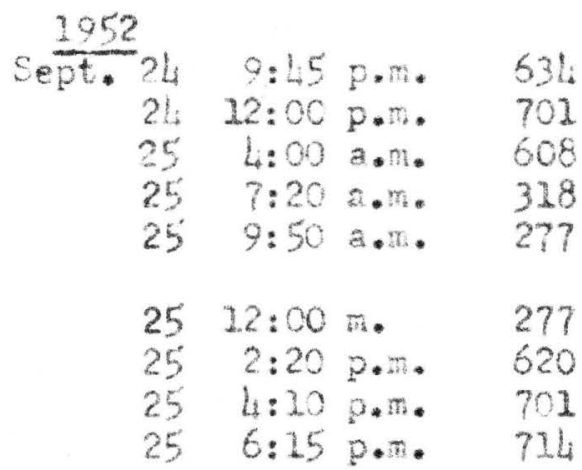

$$
\begin{array}{r}
-.58 \\
-.37 \\
-.30 \\
-.58 \\
-.64 \\
-.52 \\
-.66 \\
-.50 \\
-.10
\end{array}
$$

3,950
450
57
19
15
4,140
216
2,200
61

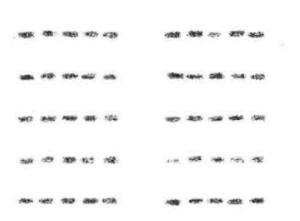

$-\infty-\infty$

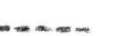

$+\infty .+$

$$
1953
$$

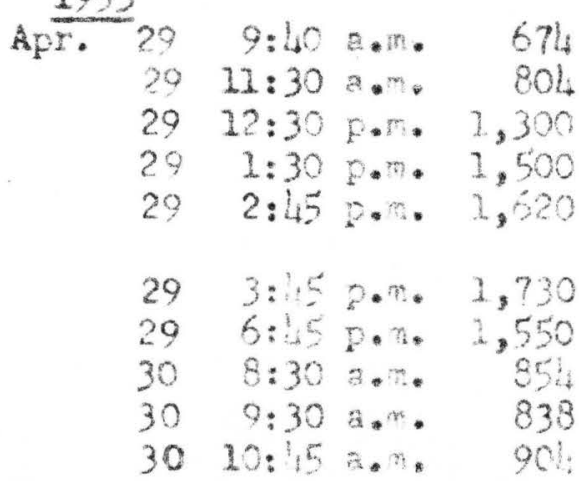

$$
\begin{array}{r}
.00 \\
-.40 \\
-.80 \\
-.50 \\
-1.00 \\
-.90 \\
-1.1 .0 \\
-.90 \\
-.90 \\
-.80
\end{array}
$$

56
82
136
214
169
215
127
60
73
103
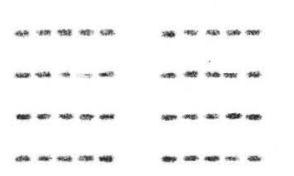

$-\infty+\infty$

$-\infty \ldots$

\begin{tabular}{|c|c|c|c|}
\hline 66 & 88 & 100 & $\ldots$ \\
\hline 64 & 25 & 100 & $-\cdots \infty$ \\
\hline 61 & 96 & 100 & $-\infty$ \\
\hline 40 & 89 & 100 & $\ldots \ldots$ \\
\hline 52 & 93 & 100 & $\cdots$ \\
\hline 39 & 85 & 96 & 100 \\
\hline 66 & 96 & 100 & $\ldots$ \\
\hline 72 & 100 & $-\infty$ & $\ldots \ldots$ \\
\hline 60 & 97 & 120 & $\ldots$ \\
\hline 60 & 96 & 100 & $\ldots$ \\
\hline
\end{tabular}

$-\infty-$

$-\infty-\infty$

$-\infty \ldots$

$-\infty-\infty$

$-\ldots$

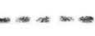

$\infty-\infty$

an-

- $-\infty-\infty$

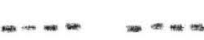

$-\infty+\infty$

$-\infty \cdots$

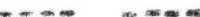

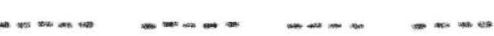

$\ldots+\cdots$

$-\infty$

n-m - $-\cdots$

103

$\begin{array}{ll}42 & 48 \\ 35 & 4 ? \\ 34 & 43 \\ 20 & 26 \\ 28 & 35 \\ 22 & 27 \\ 34 & 42 \\ 51 & 60 \\ 31 & 43 \\ 38 & 43\end{array}$


Table 3.--Observations of water discharge, suspended-aedinent, and strear-bed elavation, Plat te Rivex near Overton, Nebr.--Continued

$$
\text { Method of analysis: Sieve? }
$$

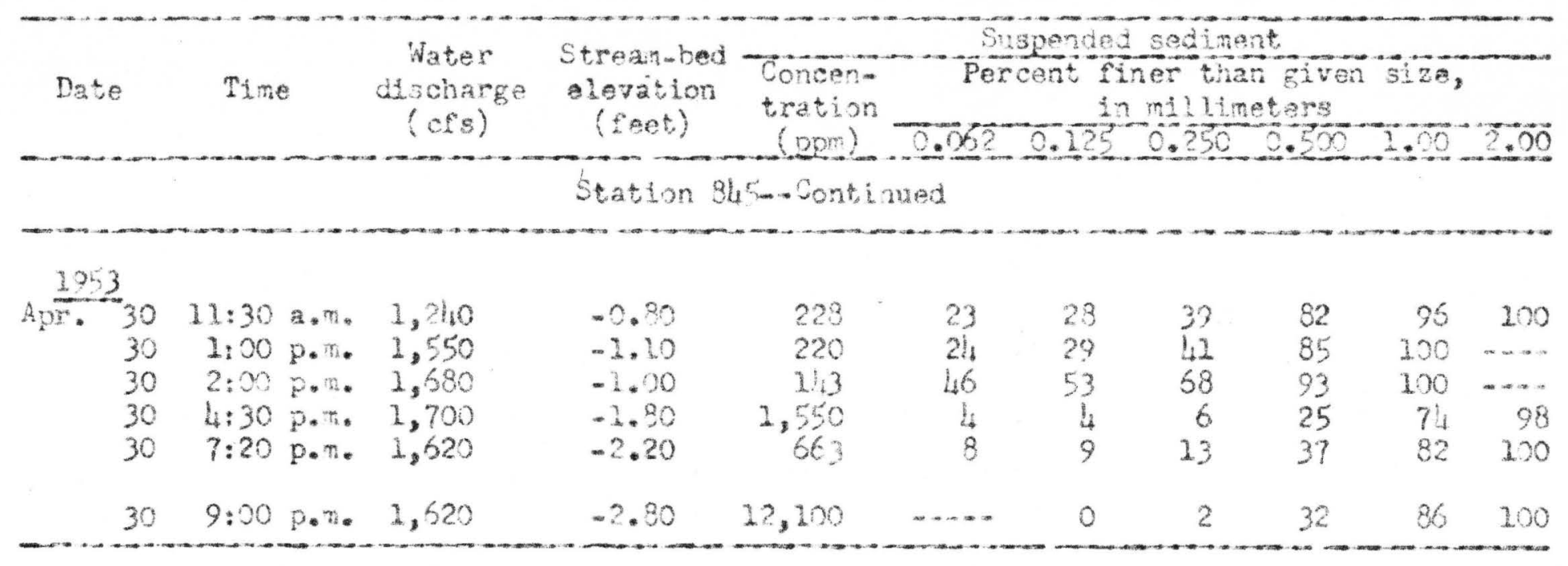


Table L.mpanticle-size gradation of bed material, Platte Hiver near Overton, Nebr.

\section{Samples colincted at the hridge or gaging section. Method of analysis: sieve?}

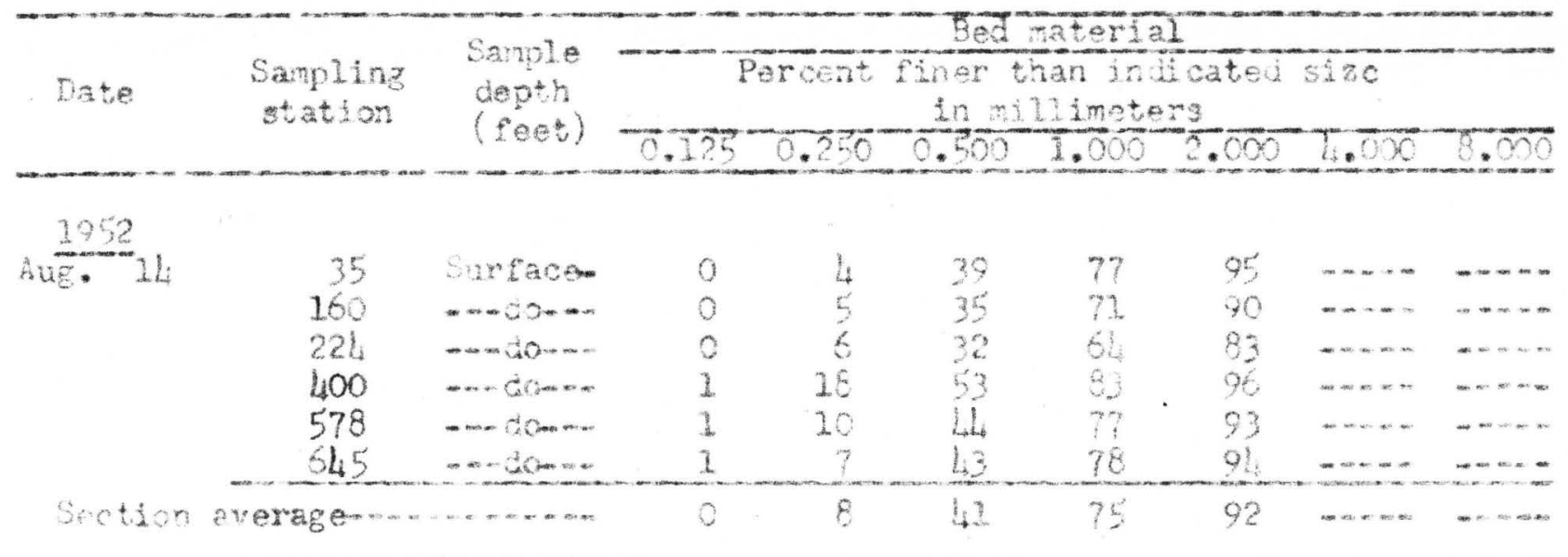

\begin{tabular}{|c|c|c|c|c|c|c|c|c|c|}
\hline sept, 25 & $\begin{array}{l}35 \\
160 \\
224 \\
400 \\
585 \\
645 \\
800\end{array}$ & 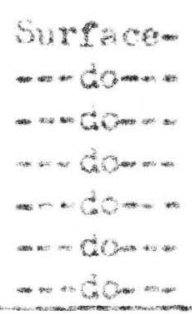 & $\begin{array}{l}0 \\
0 \\
0 \\
0 \\
0 \\
0 \\
0\end{array}$ & $\begin{array}{l}3 \\
5 \\
5 \\
5 \\
5 \\
5 \\
4\end{array}$ & $\begin{array}{l}26 \\
23 \\
29 \\
26 \\
27 \\
27 \\
29\end{array}$ & $\begin{array}{l}57 \\
46 \\
5 \\
57 \\
55 \\
58 \\
40\end{array}$ & $\begin{array}{l}81 \\
66 \\
73 \\
83 \\
78 \\
62 \\
64\end{array}$ & $\begin{array}{l}\ldots \ldots \\
\ldots \ldots \\
\ldots \ldots \\
\ldots \ldots \\
\ldots \ldots \\
\ldots \ldots \\
\ldots \ldots\end{array}$ & $\begin{array}{l}\ldots \ldots \\
\ldots \ldots \\
\ldots \ldots \\
\ldots \ldots \\
\ldots \ldots\end{array}$ \\
\hline & averag, & 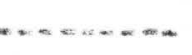 & 0 & 5 & 25 & 52 & 75 & $-\infty x+\sin x$ & $-\ldots .$. \\
\hline
\end{tabular}


Table 4.wParticlensize gradation of bed material, Platte River near Overton, Nebr..-Continued

Samples collected at the bridge or gaging section. Mathod of aralysis: 5 ter 0

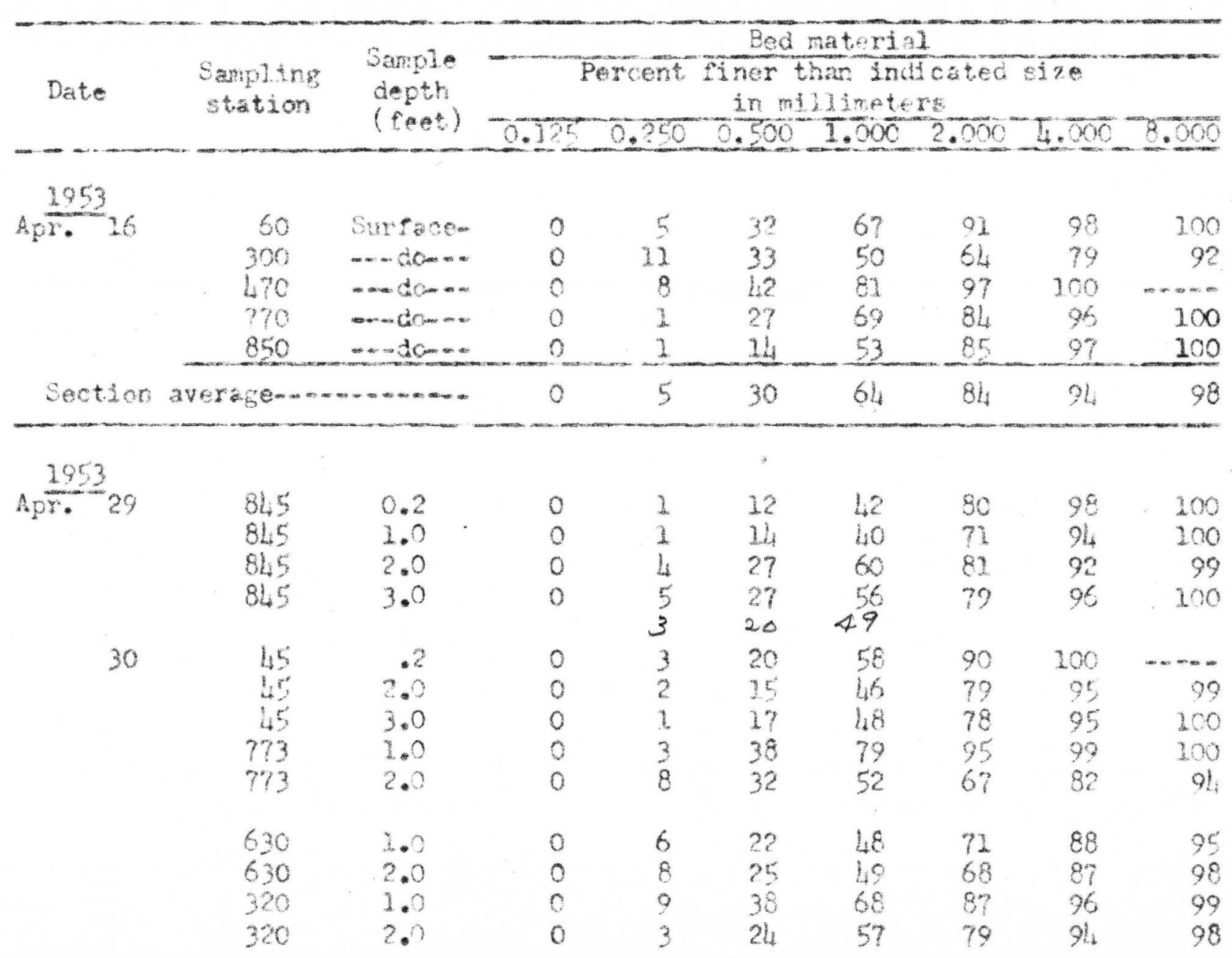


Table 4.-.Particle-size gradation of bed material, Platte River near Overton, Nebr.--Continued

$$
\text { Samples collected at the brige or gaging section. Method of }
$$
anelysis: sieve?

\begin{tabular}{|c|c|c|c|c|c|c|c|c|c|}
\hline & & & & & Bed & aterd & & & \\
\hline Date & $\begin{array}{l}\text { Sampling } \\
\text { station }\end{array}$ & Sample & & ecort & $\begin{array}{l}\text { Iner } \\
\text { in } \mathrm{m}\end{array}$ & inet & ated & size & \\
\hline & & (feet) & 0.12 & 0.250 & 0.200 & .000 & .000 & 1.000 & 3.000 \\
\hline 1953 & & & & & & & & & \\
\hline $\operatorname{sen}^{2} \cdot 24$ & 865 & Surface- & 0 & 2 & 30 & 69 & 92 & 99 & 100 \\
\hline & 845 & -ndom $=$. & O & 3 & 24 & 59 & 86 & 98 & 100 \\
\hline & 800 & $m-16=\cdots$ & 0 & 8 & 35 & 59 & 79 & 92 & 100 \\
\hline & 750 & $-2 \mathrm{shm}-m$ & 1 & 17 & 74 & 97 & 99 & 100 & $-\infty-\infty$ \\
\hline & 520 & $n-d 0=-n$ & 0 & 9 & 46 & 77 & 91 & 100 & - \\
\hline & 340 & $-m-400=$ & 0 & 6 & 30 & 57 & 79 & 93 & \\
\hline & 130 & $\cdots$ dom-n. & 0 & 7 & 38 & 68 & 93 & 100 & 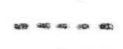 \\
\hline & 140 & $m-\mathrm{dom}=$ & 0 & 1 & 12 & 54 & 87 & 98 & 200 \\
\hline Section & average $\ldots . .$. & $-\ldots+\infty+\infty \cos x=$ & $c$ & 7 & 36 & 68 & 88 & 98 & 100 \\
\hline Mean oi & four se & 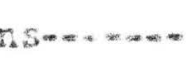 & 0 & 6 & 33 & 65 & 85 & $-\infty+\infty$ & \\
\hline
\end{tabular}


Table 5.--Periodtc observations of water discharge and water-3urfoce slope, Platte ilver near Overton, Nebr.

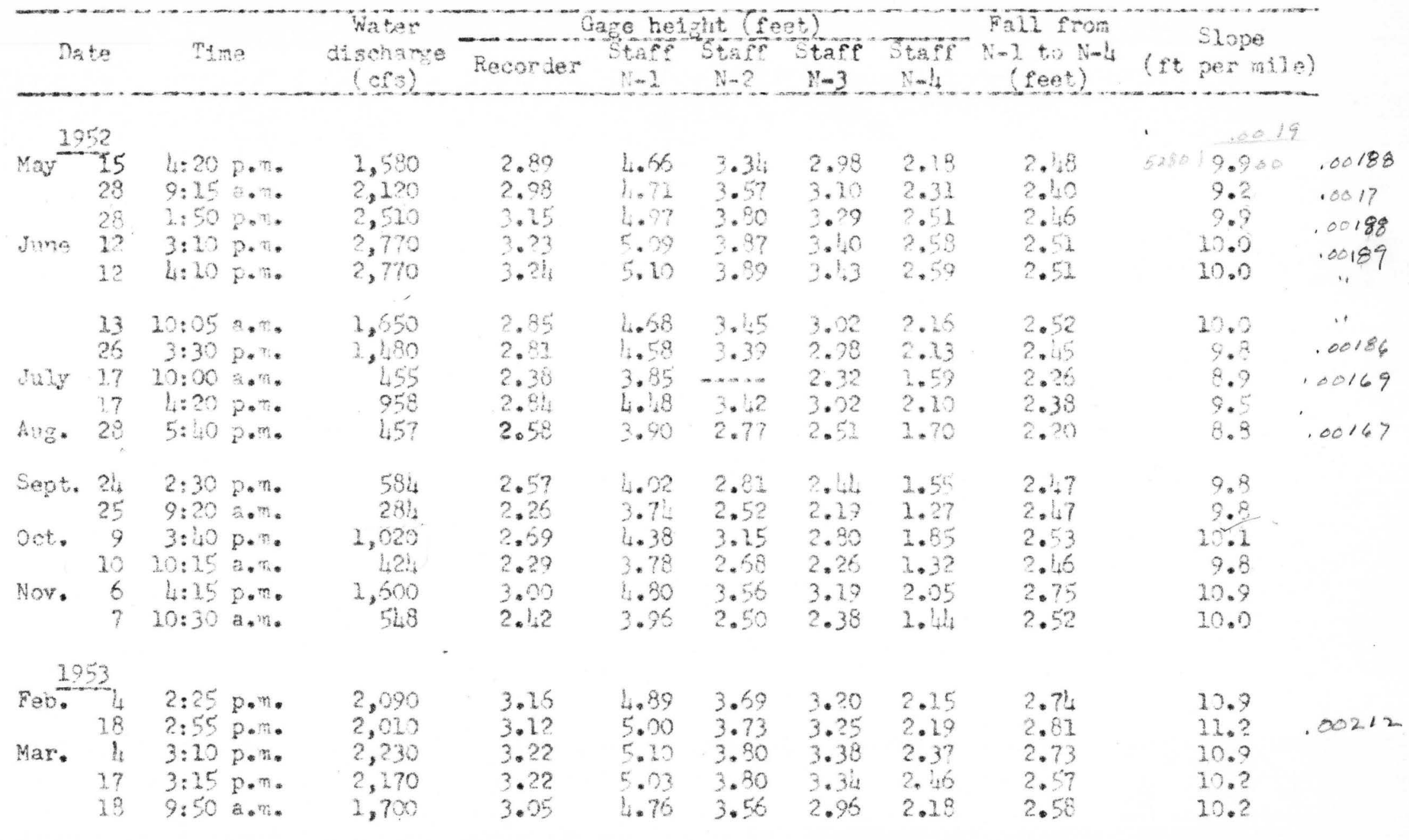


Table 5.-mperiodic observations of water discharge and water-3umace slope, Placte River near Overton, Nobr.-.Continued

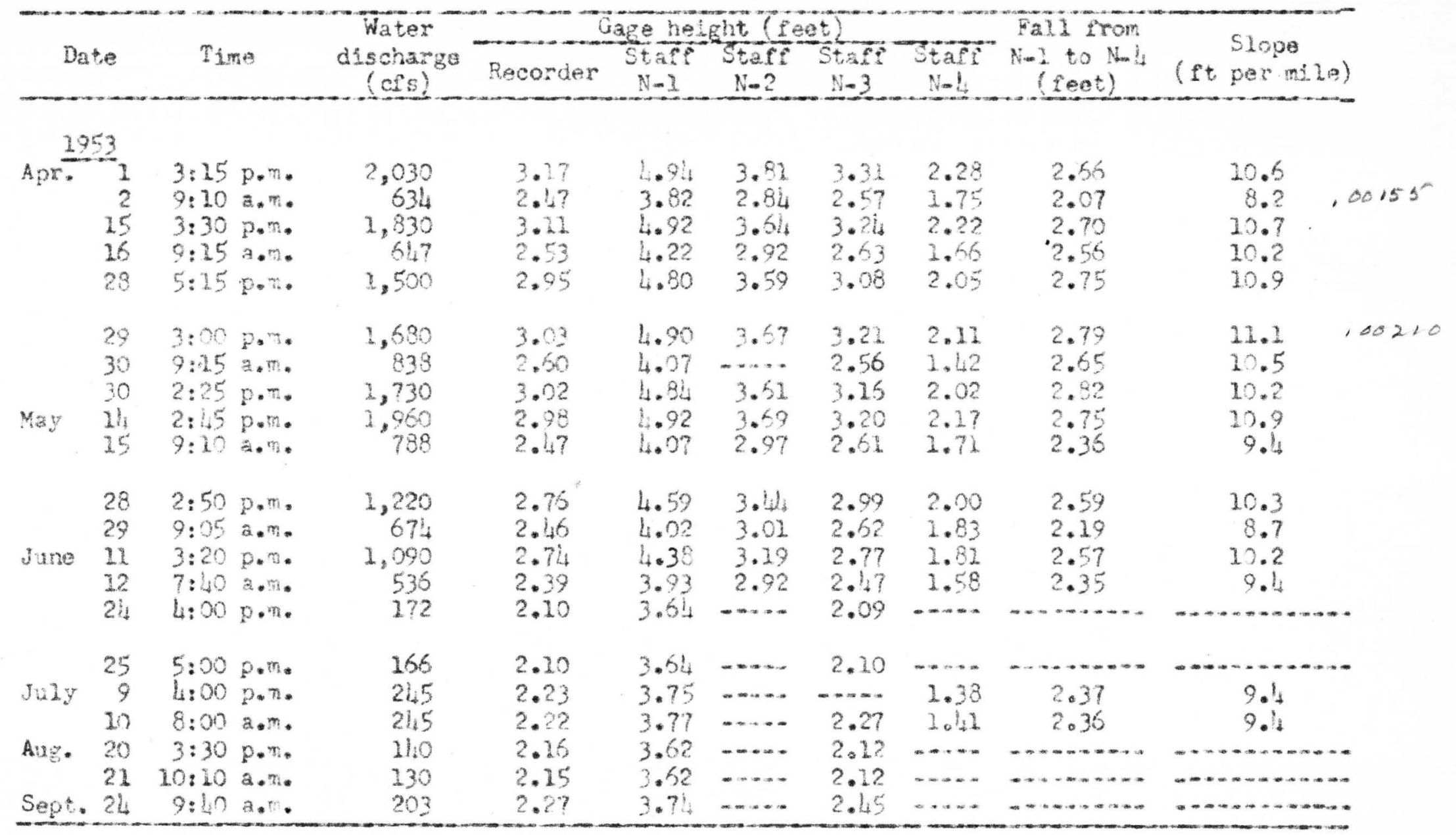


Table 6.-Comparison of total sedinent dische rge computed by the modified Einstein procedure with neasured sediment discharge, Platte Kiver near Overton, Nebr.

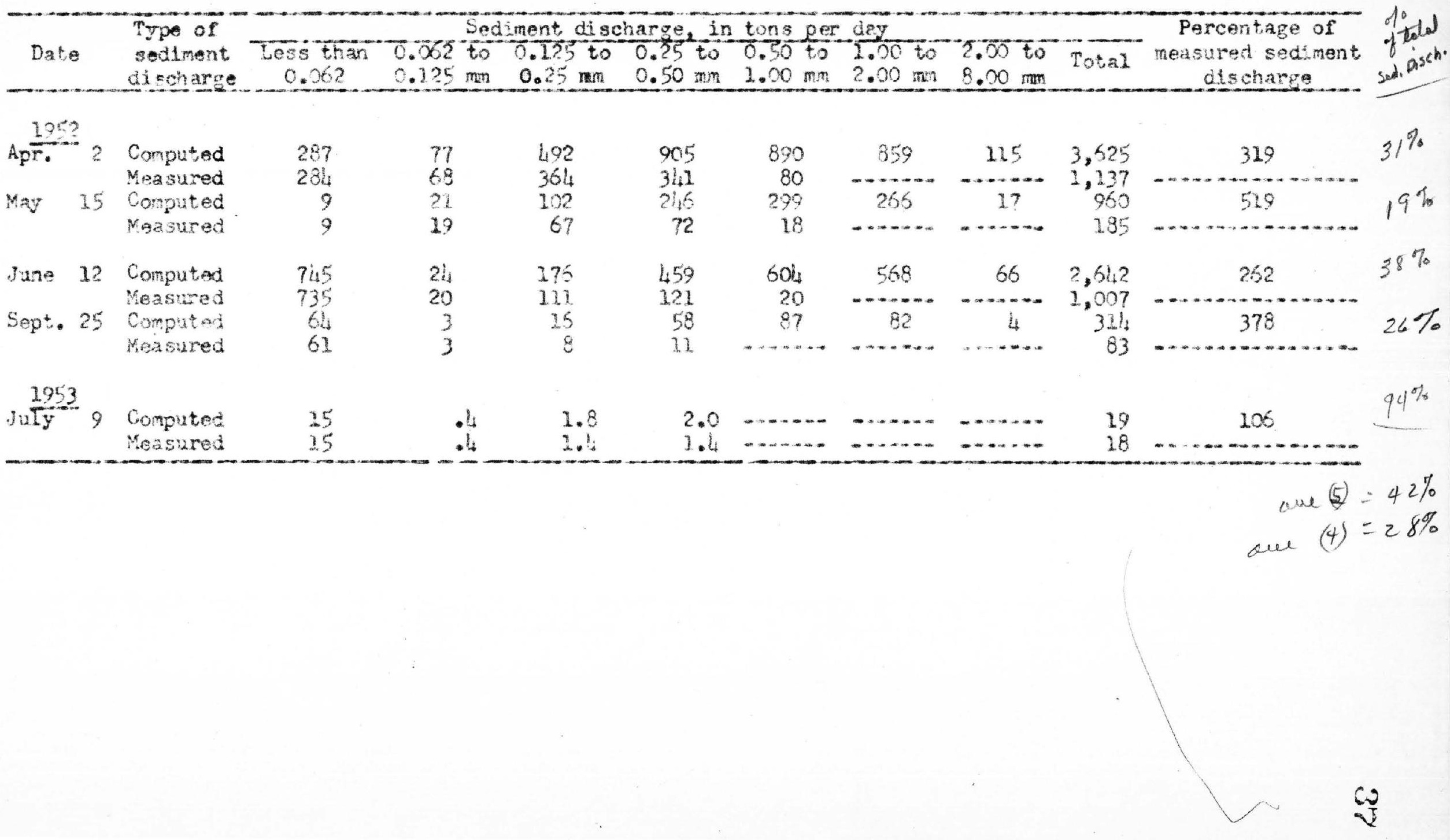

